\title{
Colours of minor bodies in the outer solar system
}

\section{A statistical analysis revisited ${ }^{\star}$}

\author{
O. R. Hainaut ${ }^{1}$, H. Boehnhardt ${ }^{2}$, and S. Protopapa ${ }^{3}$ \\ ${ }^{1}$ European Southern Observatory (ESO), Karl Schwarzschild Straße, 85748 Garching bei München, Germany \\ e-mail: ohainaut@eso.org \\ 2 Max-Planck-Institut für Sonnensystemforschung, Max-Planck Straße 2, 37191 Katlenburg-Lindau, Germany \\ 3 Department of Astronomy, University of Maryland, College Park, MD 20 742-2421, USA \\ Received 9 May 2012 / Accepted 27 July 2012
}

ABSTRACT

\begin{abstract}
We present an update of the visible and near-infrared colour database of Minor Bodies in the Outer Solar System (MBOSSes), which now includes over 2000 measurement epochs of 555 objects, extracted from over 100 articles. The list is fairly complete as of December 2011. The database is now large enough to enable any dataset with a large dispersion to be safely identified and rejected from the analysis. The selection method used is quite insensitive to individual outliers. Most of the rejected datasets were observed during the early days of MBOSS photometry. The individual measurements are combined in a way that avoids possible rotational artifacts. The spectral gradient over the visible range is derived from the colours, as well as the $R$ absolute magnitude $M(1,1)$. The average colours, absolute magnitude, and spectral gradient are listed for each object, as well as the physico-dynamical classes using a classification adapted from Gladman and collaborators. Colour-colour diagrams, histograms, and various other plots are presented to illustrate and investigate class characteristics and trends with other parameters, whose significances are evaluated using standard statistical tests. Except for a small discrepancy for the $J-H$ colour, the largest objects, with $M(1,1)<5$, are indistinguishable from the smaller ones. The larger ones are slightly bluer than the smaller ones in $J-H$. Short-period comets, Plutinos and other resonant objects, hot classical disk objects, scattered disk objects and detached disk objects have similar properties in the visible, while the cold classical disk objects and the Jupiter Trojans form two separate groups of their spectral properties in the visible wavelength range. The well-known colour bimodality of Centaurs is confirmed. The hot classical disk objects with large inclinations, or large orbital excitations are found to be bluer than the others, confirming a previously known result. Additionally, the hot classical disk objects with a smaller perihelion distance are bluer than those that do not come as close to the Sun. The bluer hot classical disk objects and resonant objects have fainter absolute magnitudes than the redder ones of the same class. Finally, we discuss possible scenarios for the origin of the colour diversity observed in MBOSSes, i.e. colouration caused by evolutionary or formation processes.

The colour tables and all plots are also available on the MBOSS colour web page ${ }^{\star \star}$, which will be updated when new measurements are published
\end{abstract}

Key words. Kuiper belt: general - comets: general - techniques: photometric - astronomical databases: miscellaneous methods: statistical

\section{Introduction}

Minor bodies in the outer solar system (MBOSSes) comprise objects in the Kuiper belt (KB) and more generally in the trans-Neptunian (TN) region, as well as small bodies in the giant planets region that came from either the $\mathrm{KB}$ or $\mathrm{TN}$ regions, but are now no longer immediate members of these environments. Centaurs are considered as escapees (Gladman et al. 2008; Kavelaars et al. 2008), scattered from the KB and TN environments towards the Sun, possibly also representing a major source of short-period comets (SPCs). Some MBOSSes may also be found among the satellites of the giant planets since they might have been stranded there by the gravitational capturing from the Centaur population (Duncan \& Levison 1997). Another large population of MBOSSes can be found as longperiod (LPCs) or Oort cloud comets. These objects are believed to have formed in the region of the giant planets and been scattered afterwards into the very distant domains of the solar

^ Full Tables 2 and 3 are only available at the CDS via anonymous ftp to cdsarc.u-strasbg.fr (130.79.128.5) or via

http://cdsarc.u-strasbg.fr/viz-bin/qcat?]/A+A/546/A115

$\star \star$ http://www.eso.org/ ohainaut/MBOSS system (Dones et al. 2004), although it cannot presently be excluded that contamination by extra-solar comets (Levison et al. 2010) may exist among the Oort Cloud population.

The MBOSSes found in the planetary system environment formed and have evolved in the region of the giant planets. They are considered as remnants of the planetesimal population, but their current distance range is not necessarily that of their formation. Nonetheless, MBOSSes may contain valuable information about the environment and the physical conditions at the time of their formation. On the other hand, their prolonged presence in the region of the distant planets and beyond may have caused alterations to their physical properties. For instance, high energy and particle radiation is suspected to modify the colours and albedos of materials in space (de Bergh et al. 2008) and collisions can affect the body either as a whole (fragmentation or growth) or in parts (resurfacing with excavated material). In addition, it cannot be excluded that intrinsic activity may have changed the surface constitution of the MBOSSes, since a number of ices, believed or known to be present in these objects, can sublimate at very large distances from the Sun (for instance $\mathrm{CH}_{4}, \mathrm{CO}$, and $N_{2}$ up to about 45,65 , and $80 \mathrm{AU}$, respectively; Delsemme 1982; Meech \& Svoren 2004). Last, but 
not least, larger MBOSSes may have experienced some alteration to their internal structure (McKinnon et al. 2008). It is thus of interest to characterize the population properties and explore possible connections with the origin of the bodies and/or their evolutionary pathways in the solar system.

With this study, we focus on the photometric properties of MBOSSes and the characterization of the different dynamical populations among them. The photometric properties comprise published measurements of their photometric brightnesses, filter colours, and spectral slopes of reflected continuum light in the visible and near-infrared (NIR) wavelength regions (Doressoundiram et al. 2008). The brightness of an object is a first indicator on its size and albedo; filter colours allow a coarse characterization of the spectral energy distribution of its reflected light and may provide constraints on its surface properties, i.e. its wavelength-dependent reflectivity.

The visible and NIR wavelength region up to $5 \mu \mathrm{m}$ contains surface-reflected sunlight, i.e. essentially either a bluish, neutral, or a reddened solar spectrum, occasionally with imprinted absorption features from specific surface materials. Continuum colours and gradients are well defined, perhaps with the exception of the HKLM bands for objects with very strong ice absorption features (Trujillo et al. 2011). The photometric parameters of MBOSSes and their correlations with then dynamical and other properties of the bodies were analysed in the past using various different data-sets and statistical methods (for a review, see Doressoundiram et al. 2008, and references therein). The key findings were that the trans-Neptunian objects (TNOs) cover a wide range of colours and spectral slopes in the visible from slightly bluish $(-5$ to $-10 \% / 100 \mathrm{~nm})$ to very red (40 to $55 \% / 100 \mathrm{~nm}$ ), while in the NIR they display a fairly narrow dispersion around solar colours. Differences in the wavelength-dependent surface reflectivity were noted for dynamically hot and cold classical disk objects (CDOs), with the latter representing a very red population in the solar system. Among the hot CDOs, an anti-correlation with high significance between the surface reddening on one hand and inclination and eccentricity on the other was interpreted as an indicator of evolutionary changes due to impacts and cosmic radiation (Trujillo $\&$ Brown 2002; Peixinho et al. 2008). Centaurs may have a bimodal colour distribution with a neutral to slightly red and a very red sub-population (Tegler et al. 2008). A number of weaker correlations between photometric and dynamical properties of Kuiper belt objects (KBOs) are addressed in the literature. However, no clear convincing picture with quantitative modelling results has evolved, although various attempts to obtain a qualitative understanding of how the surface colours were produced as the result of evolutionary processes at the surface in the outer space environment have been published (for a brief review, see Doressoundiram et al. 2008).

A completely independent approach to MBOSS colour statistics was introduced by Barucci et al. (2005a), where the objects were grouped based on their $B, V, R, I, J$ colours to form taxonomic classes. The colour study was performed using a principal component (PC) analysis (Reyment \& Joreskog 1993) and with the G-mode multivariate statistics (Coradini 1977; Fulchignoni et al. 2000). Fulchignoni et al. (2008) describe in detail the method and its main results. In the PC analysis, the original $n$ colours are replaced by the minimum possible number of linear combinations of colours (the $m$ principal components) required to reproduce the original distribution, the $n-m$ additional ones containing only noise. They found that most of the MBOSS colour range is accounted for by a single variable, closely related to the overall slope of the reflectivity spectrum over the visible range (which we define quantitatively in Sect. 2.1). The G-mode analysis reveals two main classes, labeled BB and RR, containing the neutral-grey and very red objects, respectively. The intermediate objects sort themselves into two additional classes, BR and IR. Perna et al. (2010) published an updated list of the taxonomic classes for 151 objects, and studied their distributions over the dynamical classes and other parameters. In the 1970s-1980s, the G-mode-based taxonomic classification of main belt asteroids was very successful (Tholen 1984; Tholen \& Barucci 1989; Barucci \& Fulchignoni 1990), the resulting classes becoming the basis for the physico-dynamical understanding of these objects, which is also the ultimate goal of the ongoing MBOSS taxonomic classification.

In many cases, the published colour and spectral gradient analyses rely on different data-sets collected at different telescopes and instruments and different groups. The sample sizes have grown with time reaching more than 100 TNOs and Centaurs. A summarizing database of MBOSS colours comprising data from many different papers were first compiled and analysed by Hainaut \& Delsanti (2002, hereafter Paper I) and was publicly accessible through the Internet; it is still available from the MBOSS web site, but is superseded by this work.

In this paper, we provide a version of this MBOSS colour database that has been updated both in terms of populations and collected data. It can be used either for population-wide analysis, as done in this paper, or to support other works on specific objects or group of objects either relying on the averaged data presented here (e.g. to get the colour of a given object) or by going back to the original publications listed for each object. We present new statistical analyses of the MBOSS populations based on an enhanced database of photometric brightnesses and colour measurements of the objects published in the literature and applying qualified selection criteria to the data. The qualifying criterion of the data are described in Sect. 2. Section 3 introduces the statistical methods and their application goals for the MBOSS analysis and outlines the results achieved. Possible interpretations of the findings related to their formation and evolutionary pathways are discussed in Sect. 4 . The paper ends with a brief summary of both the major findings and open and controversial issues, as well as prospects for their clarification in Sect. 5.

\section{Description of the database}

The database collects photometric information about MBOSSes, namely of KBOs and in the immediate TN region, of Centaurs, of short- and long-period comets (SPCs and LPCs), and of Jupiter Trojans. It does not contain information on satellites of the giant planets as far as they are considered to be captured MBOSSes, nor of Trojans of other planets. The objects are listed under their current official designation, i.e. number and name if available, or number and provisional designations, or provisional designation only.

For objects presenting cometary activity, the measurements collected here refer to the nucleus only, not to the dust and gas component. In the case of objects with satellites, measurements for the whole system are listed under the main designation (e.g. $26308=1998$ SM165), while an individual member of the system is indicated by a suffix $(+\mathrm{B}$ for the first satellite, for instance 134340-Pluto+B refers to Charon). In the statistical analysis, the satellites are not given a special status: they just count as individual objects.

The photometric information in the database comprises magnitudes or filter colours in the UBVRIJHKL broadband filter 
system for the visible and the NIR. In rare cases also spectral gradients and/or colours measured from spectroscopic data of MBOSSes were entered, relying on the spectrum to colours conversion presented in the original paper.

All flavours of the main filters are considered together without any conversion. For instance, in this database, Bessell $R$, Kron-Cousin $R$ and $r^{\prime}$ filters are directly listed as $R$. Most authors calibrate their system using Landolt (1992), thereby naturally unifying these subtly different systems. More exotic filters are included in the database, but not used in this study.

The literature is searched for relevant papers using the SAO/NASA ADS $^{1}$, the distant EKO newsletter ${ }^{2}$, the Neese (2011) compilation of TNO and Centaurs at the Planetary Data System $^{3}$, and the astro-ph preprints from arXiv ${ }^{4}$.

Only magnitude measurements that are explicitly presented as simultaneous are considered as a single epoch. This means that the individual filters must have been observed in a sequence, so that no more than $\sim 1 \mathrm{~h}$ elapsed during a colour measurement. The only exception was for average colours obtained from full light-curves. By default, spectral gradients and colours obtained from spectroscopy fulfill the "simultaneity" criterion. Photometric measurements not obtained simultaneously are listed as separate data, and are not used for the average colour estimates. The method used to carefully combine measurements from different epochs without introducing colour artifacts from a possible lightcurve is the same as in Paper I.

The original database, described in Paper I, contained information for only 104 objects. We had neither the means to evaluate the quality of, nor the luxury to reject some of the measurements. The online version continuously grew, and the current MBOSS database includes over 550 objects, with over 2000 measurements extracted from over 100 papers. We can now afford to reject some measurements based upon a careful and quantifiable approach. The process of cleaning data should be approached with caution, as it could potentially select against special or interesting objects. We developed a method to evaluate the quality of a data-set that is robust against genuine outliers. As first noted by Boehnhardt et al. (2001) and discussed later in the present paper, most objects have a fairly linear reflectivity spectrum over the visible wavelength range; we used this to assess the quality of a set of measurements. For each individual measurement, the distance between the data point in a colour-colour diagram and the reddening line (locus of the objects with a linear reflection spectrum, see Sect. 2.3) was computed as follows. We defined $(C x, C y)$ to be the colours of the object, and $(C x, F y)$, $(F x, C y)$ the two points on the reddening line that have the same $x$ and $y$ as the data point, respectively. The distance estimator was defined as

$d=\sqrt{\sum_{i=1}^{n} \frac{\left(C_{i}-F_{i}\right)^{2}}{n}}$,

where the $C_{i}$ and $F_{i}$ terms are the coordinates in the $B-V / V-R$ and $V-R / R-I$ colour-colour diagrams where available, and $n$ the number of coordinates available ( 2 or 4$)$. The overall quality estimator for a data-set is

$D=\sqrt{\sum_{k=1}^{N} \frac{d_{k}^{2}}{N}}$,

\footnotetext{
1 http://adswww.harvard.edu

2 http://www.boulder.swri.edu/ekonews

3 http://sbn.psi.edu/pds/resource/tnocencol.html

4 http://arxiv.org/archive/astro-ph
}

where $d_{k}$ are the individual distances and $N$ the number of measurement sets in the considered paper. $D$ behaves like an error in the photometry, expressed in magnitudes. Each of the papers with $D>0.25$ were scrutinized - this threshold was selected visually as a $2 \sigma$-like limit in the plot. The faintest objects were flagged out and $D$ re-estimated. If $D$ remained above 0.25 , the process was iterated. In some cases, this brought $D$ below 0.25 , and the remaining measurements were retained, assuming that those rejected were affected by low signal-to-noise ratio. In other cases, the dispersion in the measurements was uncorrelated with magnitude and the full data-set was rejected; we consider that a problem affected that whole paper. This was the case for some of the early literature on MBOSS photometry. Before rejecting a data-set or a measurements, the colours were compared with other measurements of the same object, if available, so as to preserve objects with intrinsic unusual spectra. In addition, the majority of the objects have linear spectra, so even a few objects with non-linear spectra did not strongly affect the overall $D$ estimator of a paper (thanks to the quadratic average), and these objects were then preserved in the database.

This method obviously worked only for measurements in the visible range. Among the 107 papers considered, 33 did not have suitable data, and 7 were globally rejected. For each data-set (including IR-only papers), outliers were individually considered. Only the points that we have a strong reason to believe were affected by a problem were rejected (for instance where a note in the paper reported a problem). In addition, some papers that concentrated on objects with exceptional spectra were obviously preserved. We believe that this statistical approach to the cleaning process with a careful and conservative a posteriori assessment ensures that the global data-set is of higher quality.

The database presented in this paper is available online ${ }^{5}$, together with all the plots related to this static version of the database, as a reference. In parallel, as the database is continually evolving with the addition of new measurements, another up-to-date version ${ }^{6}$ is available, with all plots and tables. The former should be used only in direct reference to this paper, while the dynamically updated version can be used for further generic studies of MBOSSes. The updated versions are produced in exactly the same way as the static one presented in this paper, with additional objects and measurements processed as described above.

\subsection{Content of the database}

Each record internally lists the object designation as in the original publication (i.e. typically using the temporary designation for early papers, and the final number in later papers). These were converted to the current official designation, i.e. either number and name when available, number and provisional designation, or only provisional designation.

The internal database lists the measurements for each epoch. One record is constituted by the name of the target, the epoch of the measurements, the reference to the original paper, and the list of measurements for that epoch as they appear in the paper, i.e. as magnitudes, colours, or a combination of both.

The orbital elements were retrieved from the MPCORB file, which is regularly updated from the Minor Planets Center (MPC) website ${ }^{7}$. These elements were used to compute the position of the object at the epoch of the observations (if available).

\footnotetext{
5 http://WWW . eso.org/ ohainaut/MBOSS/MBOSS2 . dat

6 http://Www. eso.org/ ohainaut/MBOSS

7 http://www . minorplanetcenter.net/iau/Ephemerides
} 
The orbit semi-major axis $a$, perihelion $q$, eccentricity $e$, inclination $i$, orbital excitation defined as $\mathcal{E}=\sqrt{\sin ^{2} i+e^{2}}$, and the helio- and geocentric distances $r$ and $\Delta$ as well as the solar phase $\alpha$ at the time of the observations were stored with the individual measurements.

The measurements were averaged as in Paper I: For a given epoch, the matrix of all possible colours was populated from the magnitudes and colours available at that epoch - but without mixing different epochs. The average colours for an object were then obtained as a weighted average of the corresponding colours, using $1 / \sigma$ as the weight, where $\sigma$ was either the reported or propagated error in the individual colour measurements.

Additionally, for each epoch, we converted the $R$ magnitude (either the one reported, or obtained from another magnitude together with the corresponding colour index) into an absolute $M(1,1, \alpha)$ magnitude with the computed $r$ and $\Delta$ using $M(1,1, \alpha)=R-5 \log (r \Delta)$. As in Paper I, we neither made no assumption about the solar phase function, nor corrected for the solar phase effect. The values of $M(1,1, \alpha)$ obtained for different epochs were then averaged into a final absolute $R$ magnitude, which is reported as $M(1,1)$ in Table 2 . The solar phase effects were not considered. The observations were obtained at small solar phase angles because of the distance of the objects, and because most of the observations were acquired close to opposition. The colours of the objects, whose study is at the core of this paper, were even less affected than the absolute magnitude. We also did not make any assumption about the albedo of the object, hence did not convert the absolute magnitudes into diameters.

For each object, the slope of the (very low resolution) reflectivity spectrum obtained from the average colour indexes (Jewitt $\&$ Meech 1986) was computed as in Paper I, via a linear regression over the $B-V-R-I$ range (assigning a lower weight to the $B$ reflectivity), where most objects display a linear reflectivity spectrum (Boehnhardt et al. 2001). This gradient, $\mathcal{S}$, is expressed in percent of reddening per $100 \mathrm{~nm}$.

\subsection{Physico-dynamical classes}

Since the publication of Paper I, the understanding of the dynamical classes in the outer solar system has greatly progressed. Paper I used fairly arbitrary definitions of the dynamical classes; moreover, the fairly small number of objects in each class did not allow for a fine-grained classification. In this paper, we used the dynamical classes defined by Gladman et al. (2008) (the now so-called SSBN08 classification, from the book in which it is published). The membership of the object was allocated using a combination of integration of the orbit over time together with cut-off in the orbital element space.

For this paper, we relied on the membership list published by Gladman et al. (2008), to which about 200 objects had been added from a list generated by C. Ejeta and H. Boehnhardt (priv. comm.) in the context of the Herschel Key Program "TNOs are Cool" (Müller et al. 2009). This list was completed by the MPC catalogue of Jupiter Trojans ${ }^{8}$.

For the objects not included in these lists, we determined the dynamical class using a simplified method: objects with an obvious designation (LPCs, SPCs) were first flagged as such, then for the remaining few objects (only 26 objects in the current database), we used an algorithm based only on the osculating orbital elements, without integration of the orbit, directly inspired

\footnotetext{
8 http://wWw . minorplanetcenter.net/iau/lists/ JupiterTrojans.html
}

Table 1. Object physico-dynamical classes (see text for the definitions) and number of objects in each class, and some statistics about the overall database.

\begin{tabular}{lc}
\hline \hline Class & Number \\
\hline Jupiter Trojans & 80 \\
Resonant (3:2) & 47 \\
Resonant (others) & 28 \\
Long-period comets (LPCs) & 14 \\
Short-period comets (SPCs) & 136 \\
Centaurs & 35 \\
Scattered disk objects (SDOs) & 30 \\
Detached disk objects (DDOs) & 28 \\
Cold classical disk objects (cold CDOs) & 89 \\
Hot classical disk objects (hot CDOs) & 68 \\
\hline Database & 555 \\
\hline Objects & 2045 \\
Epochs & 100 \\
Papers
\end{tabular}

by the flow-chart Fig. 1 in Gladman et al. (2008). Furthermore, we separated the classical TNOs between dynamically hot classical disk objects (with $i \geq 5^{\circ}$ ) and dynamically cold classical disk objects $\left(i<5^{\circ}\right)$. The arguments for this separation of CDOs come from the analysis of their orbital properties, although the first indications of different sub-populations among the classical TNOs came from a photometric study of TNOs by Tegler \& Romanishin (2000) and were further evaluated in papers by Trujillo \& Brown (2002) and Doressoundiram et al. (2002). The dynamical aspects are addressed in Morbidelli et al. (2008a) and Gladman et al. (2008). We chose an inclination of $5^{\circ}$ for the separation of the two populations. A split around orbital excitation $\mathcal{E}=0.12$ gave similar results (only 3 objects among the 89 cold classical TNOs changed class). Clearly, a sharp cut in $i, e$, or $\mathcal{E}$ space is a simplification, since it can be expected that both the cold and hot populations actually partially overlap. Following Gladman et al. (2008), we also distinguished TNOs as scattered disk objects (SDOs) or detached disk objects (DDOs). The sharp cuts in orbital element space can again only be seen as an approximation, since the actual boundary between the classes is expected to have a "complicated, fractal structure" (Morbidelli et al. 2008a).

The majority of "resonant" objects belongs to the class of Plutinos in 3:2 resonance with Neptune. Objects belonging to other resonances are summarized as "Res.others" objects in the analysis below. Finally, some of the SPCs with Centaur-like orbital elements, as defined in Gladman et al. (2008) are re-assigned to that class, following Jewitt (2009) and Tegler et al. (2008), namely 29P/Schwassmann-Wachmann 1, 30P/Oterma, 165P/LINEAR, 166P/2001 T4, 167P/2004 PY42 174P aka 60558 Echeclus, C/2001 M10 (NEAT), P/2004 A1 (LONEOS), and P/2005 T3 (Read).

\subsection{The database}

Table 1 lists the classes considered and the number of objects in each class. Table 2 provides examples of individual objects, listing the object identification and its dynamical class together with the main average colours, the spectral gradient $\mathcal{S}$, and the absolute $R$ magnitude $M(1,1)$. The full table is available in the online supplement.

Table 3 shows examples of the list of references per object that were included in this study; the information for all objects is given in the online supplement. 
O. R. Hainaut et al.: Colours of minor bodies in the outer solar system. II.
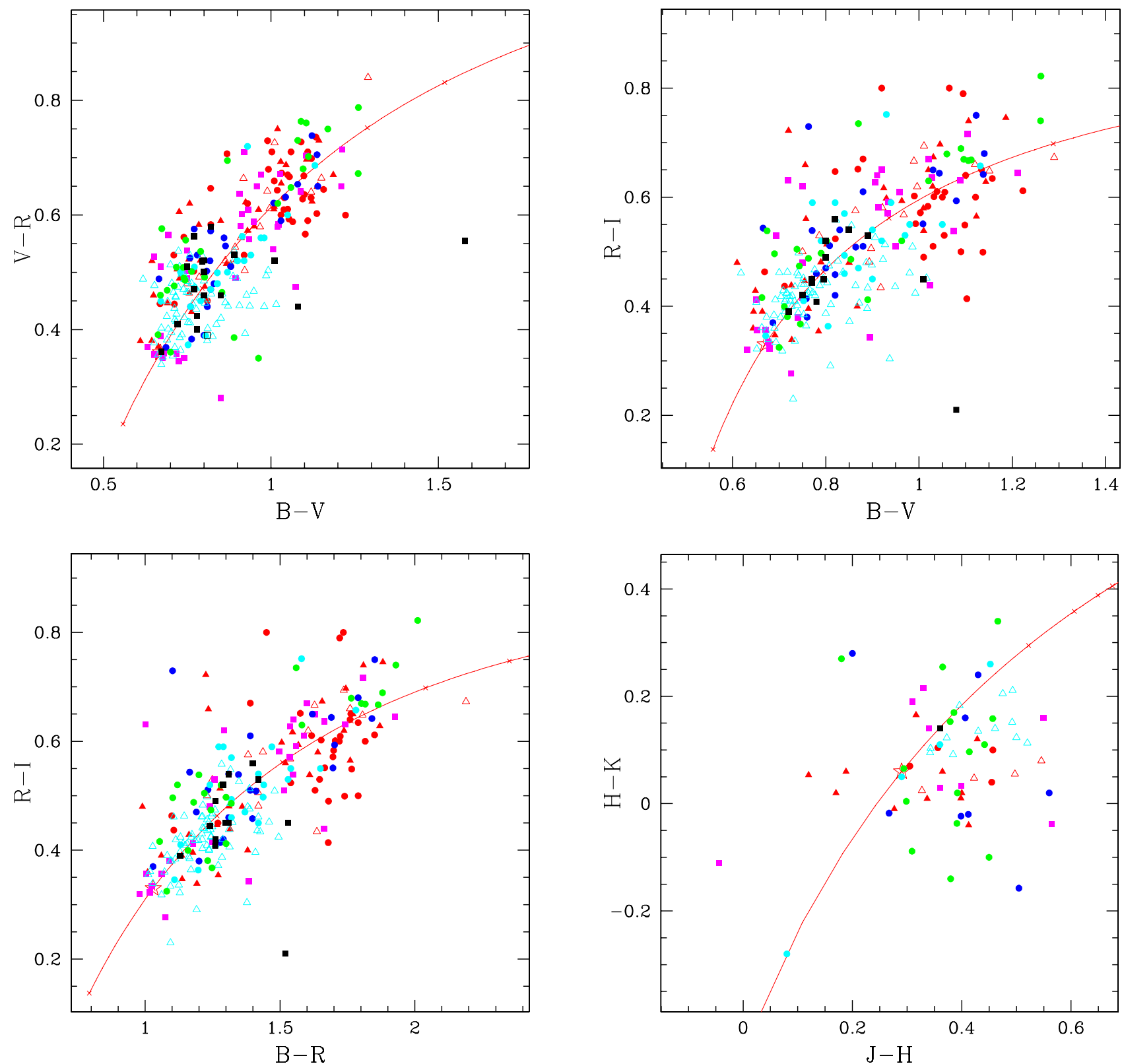

Fig. 1. Selected colour-colour diagrams of the objects. The physico-dynamical class of the objects is identified by their symbols, which are explained in Fig. 2. The red star indicates the solar colours, and the red line is the locus of objects with a flat reflection spectrum, with a small mark every $10 \% / 100 \mathrm{~nm}$. The other colour combinations are available from the MBOSS site.

Figure 1 presents some of the representative colour-colour diagrams. As a reference, the solar colours are indicated by a red star (see Paper I, Table 2 for a list of the solar colours and their references). The red "reddening line", introduced in Paper I, marks the locus of objects with a perfectly linear reflectivity spectrum over the considered colours, with a tick mark every 10 units of $\mathcal{S}$. For colour diagrams in the visible range, the distance from a data point to the reddening line therefore indicates a "bend" in the reflectivity spectrum. The symbols used indicate the dynamical class, and are explained in Fig. 2. Figure 3 shows examples of the coarse reflectivity spectra for a set of objects obtained from their filter photometry. All the other plots are available at the MBOSS site.

Figure 7 displays the spectral gradient $S$ as a function of the main orbital elements, the orbital excitation, and the $M(1,1)$ magnitude of the objects. Other similar plots, for all the colours, are available online. The symbols indicate the dynamical class.
Figure 4 shows the histogram and the cumulative distribution for an example colour and the spectral gradient. Other similar plots are available on the MBOSS web site.

\section{Results from the statistical analysis of the enhanced MBOSS database}

\subsection{Statistical tools}

Comparing the distribution by comparing their histograms "by eye" is unreliable: the size of the bins can cause artifacts or hide real features, and what appears as a strong difference can actually be of no significance. Similarly, the eye is a very powerful tool to detect alignments and clustering, even when these are insignificant.

The colour, spectral gradient and $M(1,1)$ distributions of the various MBOSS populations were therefore compared using 


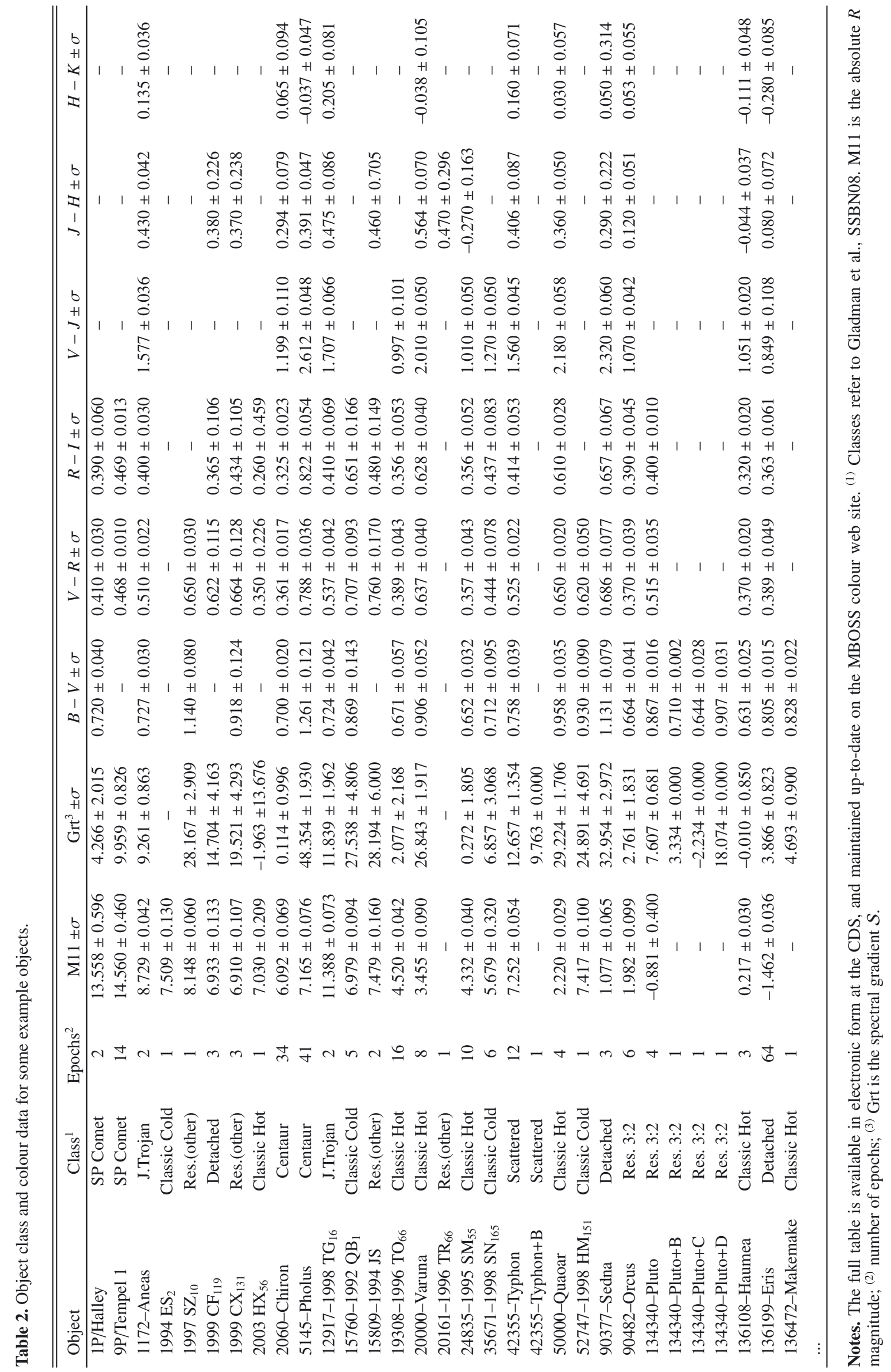


O. R. Hainaut et al.: Colours of minor bodies in the outer solar system. II.

\section{- Resonant 3:2 (Plutinos) Resonant (others) \\ Cold classic disk objects \\ Hot classic disk objects \\ - Scattered disk objects \\ Detached disk objects \\ Jupiter Trojans \\ Centaurs \\ Short-period comets \\ Long-period comets \\ Sun}

Fig. 2. Legend of the symbols used thorough this paper.

Table 3. For some objects, list of the references used, and number of epochs.

\begin{tabular}{lcc}
\hline \hline Object & References & No. \\
\hline 1994 ES 2 & Green et al. (1997) & 1 \\
7066-Nessus & Davies et al. (1998) & \\
& Romanishin \& Tegler (1999) & \\
& Bauer et al. (2003) & \\
& Davies (2000) & \\
15760-1992 QB 1 & Tegler \& Romanishin (1998) & 17 \\
& Jewitt \& Luu (2001) & \\
& Tegler \& Romanishin (2000) & \\
& Boehnhardt et al. (2001) & \\
& Benecchi et al. (2011) & \\
& Romanishin et al. (1997) & 5 \\
19308-1996 TO 66 & Jewitt et al. (2007) & \\
& Jewitt \& Luu (1998) & \\
& Romanishin \& Tegler (1999) & \\
& Hainaut et al. (2000) & \\
& Gil-Hutton \& Licandro (2001) & \\
& Davies et al. (2000) & \\
& Jewitt \& Luu (2001) & \\
& Boehnhardt et al. (2001) & \\
& Sheppard (2010) & \\
& Barucci et al. (1999) & Davies (2000) \\
& Tegler \& Romanishin (1998) & 16
\end{tabular}

Notes. The full table is available in electronic form at the CDS, and maintained up up-to-date on the MBOSS colour web site.

a set of simple statistical tests that quantifies the significance of the apparent differences, i.e. the $t$-test, the $f$-test, and the Kolmogorov-Smirnov test. The tests are described in detail in Appendix B of Paper I and references therein and can be briefly summarized as follows:

- The Student $t$-test indicates whether the mean values of the two distributions are statistically different. The implementation used here deals properly with distributions with different variances.

- The $f$-test considers whether two distributions have significantly different variances.

- Finally, the Kolmogorov-Smirnov (KS) test uses all of the information contained in the distributions (and not just their means and variances) to estimate whether they are different.
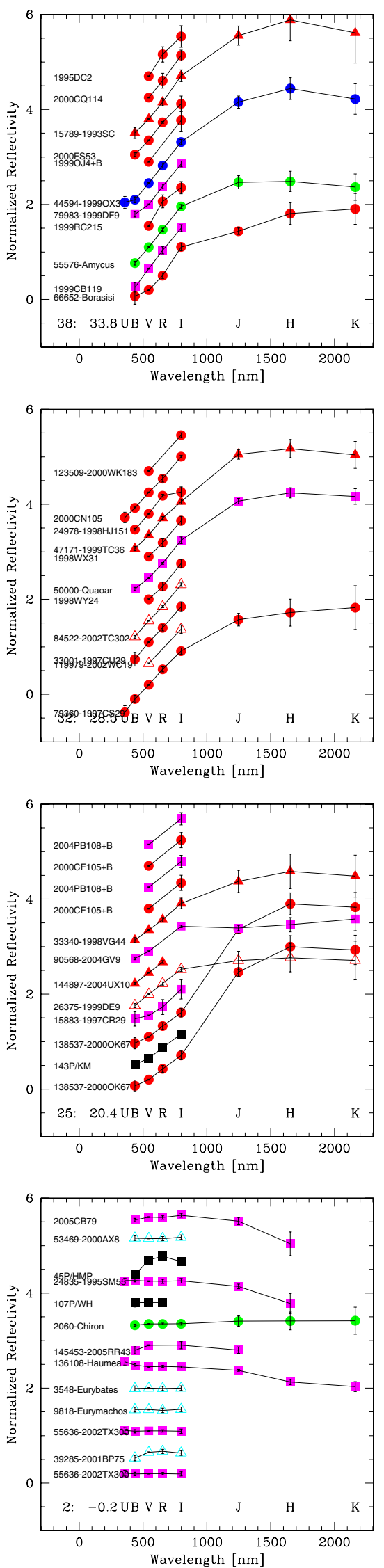

Fig. 3. Examples of reflectivity spectra for a set of objects. The reflectivity is normalized to unity for the $V$ filter, and the spectra are shifted for clarity. The physico-dynamical classes of the objects are identified by their symbols, which are explained in Fig. 2. The spectra for all the objects are available online on the MBOSS site. 

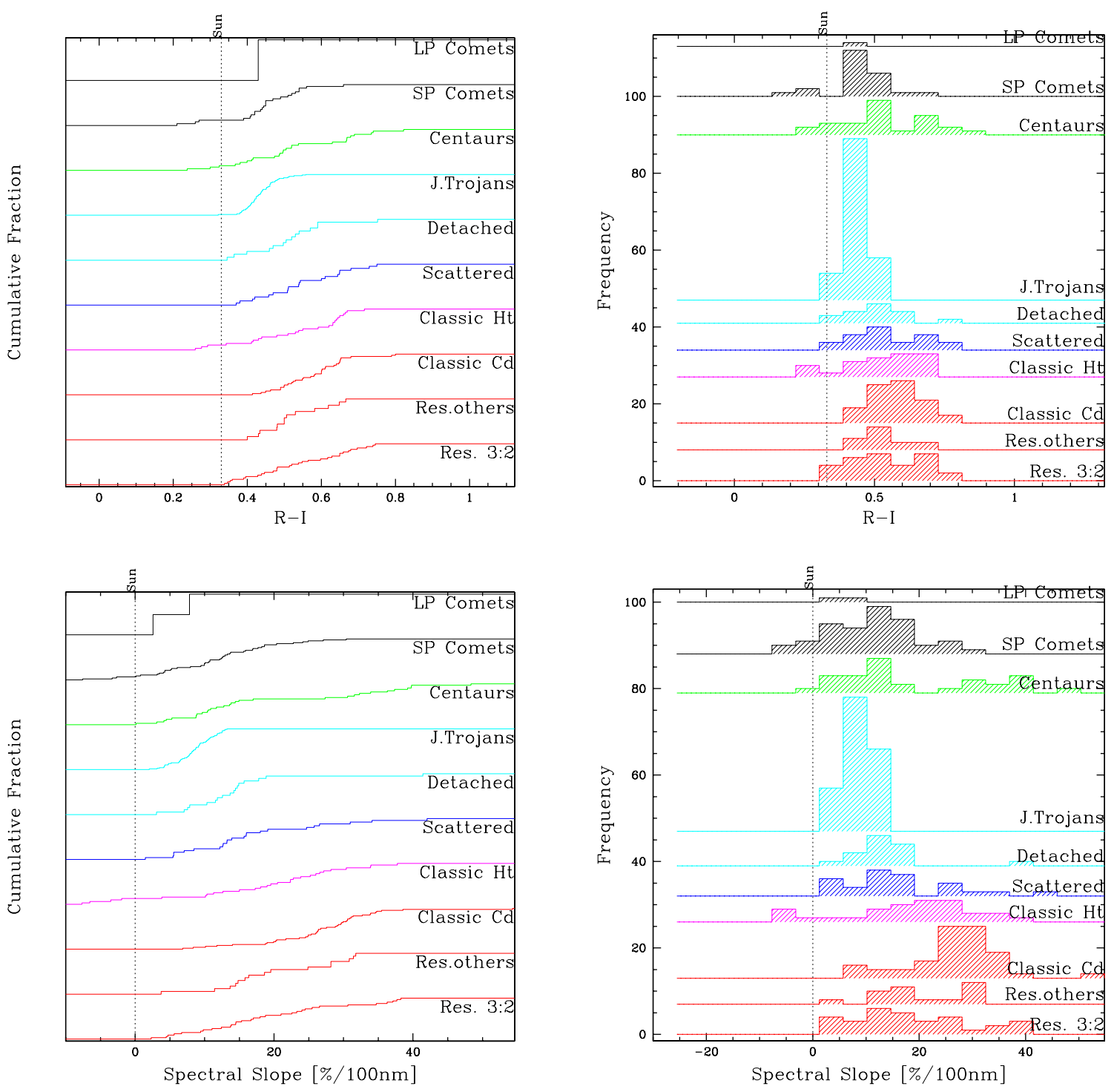

Fig. 4. $R-I$ (top) and spectral gradient (bottom) cumulative distributions (left) and histogram (right). Solar value is indicated by a dotted line. Similar plots are available on the MBOSS web site for the other colours.

For these tests to give meaningful results, the samples compared must be sufficiently large. We set the threshold at 15 units for $t$ and $f$-tests, and at 20 units for the KS test.

\subsection{The case of the brightest objects}

Large KBOs have atypical surface properties, e.g high albedo (Stansberry et al. 2008; Brown 2008), flatter spectral gradients, and $\mathrm{CH}_{4}$ ice-dominated spectra (Brown et al. 2005). For instance, Pluto is known to have a tenuous but captured atmosphere that can be re-deposited on the surface (Stern \& Tholen 1998; Protopapa et al. 2008), while (136199) Eris has an extremely high albedo (Sicardy et al. 2011) and a spectrum dominated by $\mathrm{CH}_{4}$ ice (Brown et al. 2005) and a nitrogen ice signature (Dumas et al. 2007). Schaller (2010) discusses the presence of volatiles (nitrogen, methane, and carbon monoxide) on bodies large enough to retain an atmosphere. The transition from small objects with volatile-free surfaces (such as Charon or Quaoar) to large objects with volatile-rich ones (such as Pluto) can be explained with a simple model of atmospheric escape formulated by Schaller \& Brown (2007).
We therefore decided to remove the largest objects (using their intrinsic brightness as a proxy) for some studies, so that their potentially different characteristics do not pollute the colour distributions of the other, "normal" MBOSSes. To select a cutoff value, we estimated the radius of the objects whose escape velocity $v_{\text {esc }}$ is equal to the velocity of material ejection via cometary activity $v_{\mathrm{ej}}$. The escape velocity is given by

$v_{\mathrm{esc}}=\sqrt{\frac{2 G M}{R}}$

where $G$ is the gravitational constant, $M$ the mass of the object, and $R$ its radius. Assuming a density $\rho \sim 1000 \mathrm{~kg} \mathrm{~m}^{-1}$ to be able to infer the mass from the volume of the object,

$v_{\mathrm{esc}} \sim 7.5 \times 10^{-4} R$

in IS units. To estimate the velocity of material ejection, $v_{\mathrm{ej}}$ (in $\mathrm{m} \mathrm{s}^{-1}$ ), we used the relation

$v_{\mathrm{ej}}=580 r^{-0.5} \sqrt{\frac{\mu_{0}}{\mu}}$, 
O. R. Hainaut et al.: Colours of minor bodies in the outer solar system. II.

Table 4. Averge colours and dispersions of all MBOSSes (excluding Trojans), comparing those with $M(1,1)<5$ and the others.

\begin{tabular}{lcccccc}
\hline \hline Colour & $N$ & $\begin{array}{c}\text { Aver. } / \sigma \\
M(1,1)<5\end{array}$ & \multicolumn{2}{c}{$N \begin{array}{c}\text { Aver. } / \sigma \\
M(1,1) \geq 5\end{array}$} & $t$-Prob & $f$-Prob \\
\hline$J-H$ & 22 & $0.27 \pm 0.25$ & 84 & $0.41 \pm 0.20$ & 0.025 & 0.149 \\
$H-K$ & 16 & $0.04 \pm 0.07$ & 31 & $0.07 \pm 0.12$ & 0.279 & 0.036 \\
\hline
\end{tabular}

Notes. The $q$ cut-off is set at the median value; $N$ is the number of measurements; $t$-Prob and $f$-Prob are the probabilities that the two subsamples are randomly extracted from the same distribution, as evaluated with the $t$ - and $f$-tests. The other colours show insignificant differences.

where $r$ is the heliocentric distance in $\mathrm{AU}$, and $\mu_{o} / \mu$ the ratio of the molecular mass of the species driving the activity to that of water. This relation was obtained by measuring the expansion of cometary comae, and is supported by a theoretical analysis see Delsemme (1982) for a discussion. This velocity is the terminal velocity of the gas in the case of a small comet, and it is directly controlled by the thermal velocity of the sublimating gas. For the TNOs, we used $\mu_{o} / \mu=0.64$ for CO. By equating $v_{\mathrm{ej}}$ to $v_{\text {esc }}$, we obtained a critical radius $R_{\mathrm{c}}$ above which an object is likely to retain some of the material ejected by cometary activity

where $R_{\mathrm{c}} \sim \frac{6.2 \times 10^{5}}{\sqrt{r}}$.

$R_{\mathrm{c}}$ is in $\mathrm{m}$ and $r$ in AU. Delsanti et al. (2004) discuss the evolution of $R_{\mathrm{c}}$ with different species and distance. For our purpose, it is enough to say that $R_{\mathrm{c}} \sim 150 \mathrm{~km}$ at $r=17 \mathrm{AU}$ and $100 \mathrm{~km}$ at 43 AU. Stansberry et al. (2008), based on their large set of measurements with the Spitzer Space Telescope, indicate that large TNOs have higher than average albedos. We used $p=0.2$ to convert $R_{\mathrm{c}}$ in an absolute magnitude, leading to

$M(1,1) \sim 1.9+2.5 \log r$,

again with $r$ in AU. This gives $M(1,1) \sim 5$ at $r=17 \mathrm{AU}$, and 6 at $43 \mathrm{AU}$. In what follows, we took $M(1,1)=5$ as a conservative limit, below which objects are likely to keep at least part of their atmospheres in the Centaur and TN regions, thus be potentially affected by different resurfacing processes. This choice is partly arbitrary. We performed the following analysis with different cut-off values, leading to similar results.

To verify whether we were justified in separating the bright $M(1,1)$ objects from the others, we compared their colours and gradients with those of the faint objects. The Trojans were not considered in this test, whose results are summarized in Table 4.

With the exception of the $J-H$ colour, the bright and the faint objects have compatible mean colours and overall distribution. In the infrared, the bright objects have marginally bluer colours $(J-H=0.27 \pm 0.25)$ than the faint ones $((J-H=0.41 \pm 0.20)$; this has only a probability of $2.5 \%$ of occurring by chance, i.e. this difference is marginally significant. Similarly, the bright objects have a slightly broader distribution of $H-K$ colour than the fainter ones (at the 3\% level). When performing these tests on individual or groups of classes, the result was a slightly more significant for the resonant objects, but could not be tested for the other groups, which did not have enough bright objects. Nevertheless, removing the non-resonant objects increased the significance of the result for the resonant objects. Additionally, one must consider the possibility that the larger dispersion in $H-K$ for the fainter objects is connected to the lower signalto-noise ratio in $K$, as the sky is much brighter in this band and the instruments tend to be less sensitive there. Considering the colours of the various dynamical classes in the visible wavelength range for both the bright $(M(1,1)<5)$ and faint objects, the colour distributions of the bright objects were found to be indistinguishable from those of the fainter ones, indicating that the object populations might be rather uniform despite some larger objects possibly having some different surface properties (for instance, albedo) and intrinsic activity possibly playing a role in resurfacing the bodies. We note, however, that the NIR colours of individual KBOs can be affected if a significant fraction of their surfaces is covered with ices which display strong absorptions (such as $\mathrm{CH}_{4}$ ), particularly in $H$ and $K$ bands. For example, the colours of Pluto, Eris and $2005 F Y_{9}$ (see Brown 2008) show this effect. The presence of these ice absorption lines can be inferred from the unusual negative slope of the NIR spectral gradients (i.e. from $J$ to $H$ and $K$ bands).

In summary, the infrared colours of the objects with bright absolute magnitudes differ slightly from those of the fainter objects. We therefore separated them for most colour tests, but we also re-incorporated them into some tests of the visible colours where more objects were helpful.

On the basis of these results, one might consider including the intrinsically brighter objects in the remainder of the study. However, we decided not to: if they were indeed similar, they would only marginally increase the size of the sample, without changing our conclusions. If they eventually turned out to be different, as possibly suggested by the NIR colours, we did the right thing in keeping them separate.

\subsection{Global characteristics of individual classes}

The MBOSS database that we analyse contains photometric measurements of ten different dynamical classes (see Fig. 2), i.e. the SPCs, the LPCs, the Jupiter Trojans, Centaurs, KBOs in 3:2 (Plutinos $=$ Res. 3:2) and in other orbital resonances (Res. others) with Neptune, CDOs in two flavors of dynamically "hot" and "cold" CDOs, the scattered disk objects (SDOs) and the detached disk objects (DDOs).

Selected colour-colour diagrams of MBOSSes are shown in Fig. 1. Colour distributions of the various classes of objects are displayed in Fig. 4, for a selection of colours and spectral gradients $\mathcal{S}$. At first sight, the ranges of visible colours are similar for the several dynamical groups (Plutinos and resonants, CDOs, SDOs, and Centaurs), ranging from slightly bluish $(-10 \% / 100 \mathrm{~nm})$ compared to the Sun to very red $(55 \% / 100 \mathrm{~nm})$. Jupiter Trojans do not appear to contain any very red objects (i.e. with spectral gradients $>20 \% / 100 \mathrm{~nm}$ ) as the other groups do. For LPCs, the number of measured objects (14) may be too small to provide representative results for the total population, although we note that the available data indicate that the spectral gradients are between about 0 and $10 \% / 100 \mathrm{~nm}$, in close agreement with the other groups.

The mean colours (and corresponding variances) for the various dynamical classes are listed in Table 7, top panel, using all data in the MBOSS2 database, and the values for the restricted data-set of objects with $M(1,1) \geq 5$ mag are in Table 7 , bottom panel. Many of these means are based on only a small number of objects and should be interpreted with care.

The average characteristics of the dynamical classes are also displayed as reflectivity spectra in Fig. 5. The red slope in the visible wavelength range, although different for individual groups, levels off in the NIR with transition between the $I$ and $J$ or $H$ bands. 

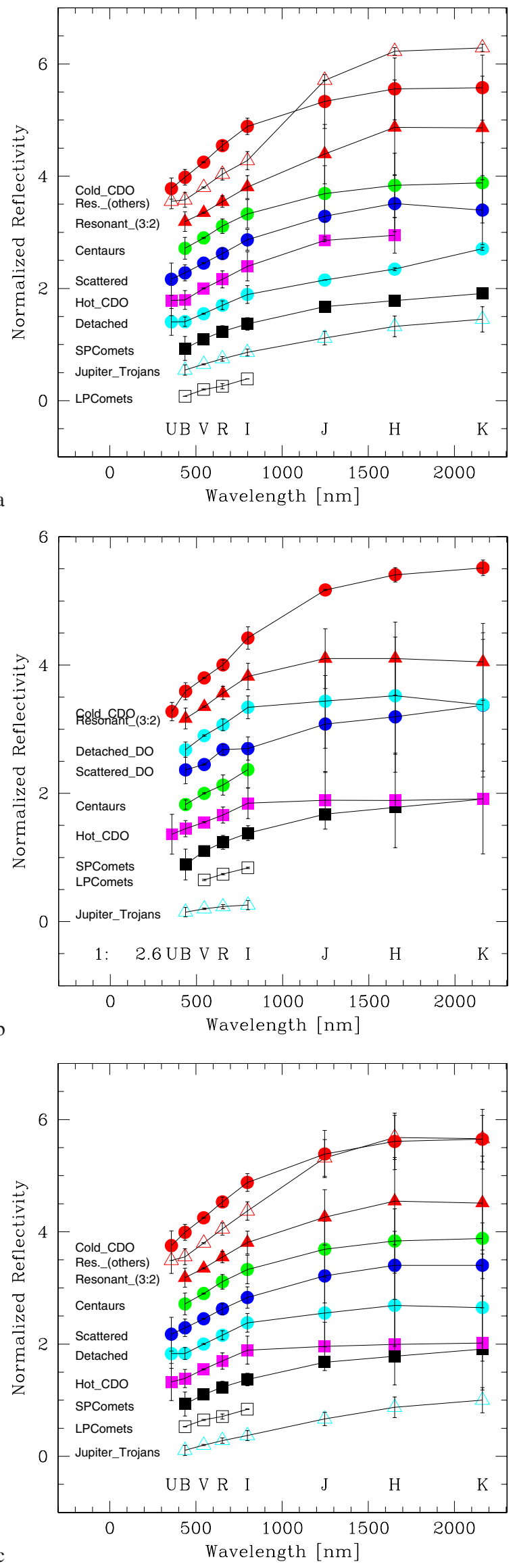

Fig. 5. Average reflectivity spectra for the different physico-dynamical classes. a) small objects $(M(1,1)>5 \mathrm{mag})$; b) large objects only $(M(1,1)<5 \mathrm{mag}) ; \mathbf{c})$ all objects.

\subsection{Comparisons between classes}

In our comparison, we considered the photometric properties of the dynamical classes all groups except LPCs, since are too sparse to expect firm conclusions.

\subsubsection{Colours and spectral gradients}

Comparing the colour distributions of the different physicodynamical classes, we can make the following observations (see Fig. 4):

- The distributions of both the colours and the spectral gradients in the visible wavelength range differ among the groups, in terms of their extent, shape and peak location. Taking the spectral gradients as an example, the peak level increases starting with the Jupiter Trojans (5-10\%/100 nm), and continuing to the SPCs, Centaurs, DDOs, SDOs, Plutinos (and possibly the resonant objects) $(10-20 \% / 100 \mathrm{~nm})$, and the hot CDOs (20-30\%/100 nm), before ending with the cold CDOs which are the reddest objects $(25-35 \% / 100 \mathrm{~nm})$ among the MBOSSes. The colour ranges and distribution width can clearly be inferred from the cumulative distribution functions (see left panels of Fig. 4).

- Relevant secondary peaks in the frequency distributions may exist for Centaurs and Plutinos at higher reddening. At least for Centaurs, statistical arguments for a bimodal surface colour distribution have been presented in numerous papers (e.g. Tegler \& Romanishin 1998, 2003; Peixinho et al. 2003; Delsanti et al. 2006; Tegler et al. 2008). Tegler et al. (2008) presented a detailed analysis of the Centaurs' bimodality, based on a sample of 26 objects, i.e. almost as large as the one presented here $(29$ objects, so we do not present a new analysis). They concluded that the $B-R$ distribution is bimodal with a confidence level of $99.5 \%$, with 10 red Centaurs and 16 gray ones. The red ones have marginally smaller orbital inclinations, and higher albedos at the $99 \%$ confidence level.

- The frequency distribution in $J-H$ instead gives a rather uniform picture for all dynamical groups with respect to colour range and peak position (with a few singular exceptions for Plutinos and CDOs).

This global characterization indicates that parameters of the visible spectral energy distribution are better diagnostics of both the global surface reflectivity and differences among MBOSSes than the NIR colours. However, we note that the NIR spectral data of MBOSSes is more sensitive to compositional differences than visible one, because stronger absorption bands, in particular of icy compounds, are found in the NIR wavelength domain (Barucci et al. 2008).

We now compare the populations using the statistical tests described in Sect. 3.1. First of all, we stress that a common feature of all the statistical tests applied is the estimate of a probability that the two populations compared are not randomly extracted from the same population. In other words, the statistical tests can very firmly establish whether two populations significantly differ. A contrario, no statistical test can prove that two populations are identical. For instance, the colour distributions of the Centaurs and Jupiter Trojans (see Table 5) are radically different with a very high significance level: it is totally improbable that these two populations were randomly extracted from the same reservoir. However, the spectral gradient distributions of SDOs and DDOs indicate that these objects could have been 
O. R. Hainaut et al.: Colours of minor bodies in the outer solar system. II.

Table 5. Statistical comparisons of the spectral gradient distributions of pairs of MBOSS populations: $t$-test, $f$-test, and KS test.

\begin{tabular}{|c|c|c|c|c|c|c|c|c|c|c|c|}
\hline & & & $\begin{array}{c}\text { Resonant } \\
\text { (others) }\end{array}$ & $\begin{array}{l}\text { Classical } \\
\text { cold }\end{array}$ & $\begin{array}{c}\text { Classical } \\
\text { hot }\end{array}$ & $\begin{array}{l}\text { Scattered } \\
\text { disk } \\
\text { objects }\end{array}$ & $\begin{array}{l}\text { Detached } \\
\text { disk } \\
\text { objects }\end{array}$ & Centaurs & $\begin{array}{l}\text { Short } \\
\text { period } \\
\text { comets }\end{array}$ & $\begin{array}{l}\text { Long } \\
\text { period } \\
\text { comets }\end{array}$ & Trojans \\
\hline$N$ & & & 15 & 43 & 28 & 23 & 17 & 26 & 44 & 0 & 60 \\
\hline \multirow{4}{*}{$\begin{array}{l}\text { Resonant } \\
3: 2\end{array}$} & 31 & $t$ & 0.701 & 0.002 & 0.713 & 0.340 & 0.056 & 0.768 & 0.006 & & 0.000 \\
\hline & & $f$ & 0.367 & 0.209 & 0.505 & 0.777 & 0.264 & 0.163 & 0.334 & & 0.000 \\
\hline & & $\mathrm{KS}$ & 0.810 & 0.000 & 0.817 & 0.612 & 0.023 & 0.438 & 0.072 & & 0.000 \\
\hline & & & Indist. & Not comp. & Indist. & Indist. & Marginal & Indist. & Marginal & $-1-$ & Not comp. \\
\hline \multirow{4}{*}{$\begin{array}{l}\text { Resonant } \\
\text { others }\end{array}$} & 15 & $\bar{t}$ & & 0.019 & 0.485 & 0.211 & 0.034 & 0.968 & 0.005 & & 0.000 \\
\hline & & $f$ & . & 0.986 & 0.166 & 0.523 & 0.881 & 0.057 & 0.810 & & 0.000 \\
\hline & & KS & & 0.019 & 0.680 & 0.183 & 0.009 & 0.101 & 0.020 & & 0.000 \\
\hline & & & & Marginal & Indist. & Indist. & Marginal & Indist. & Marginal & $-1-$ & Not comp. \\
\hline \multirow{4}{*}{$\begin{array}{l}\text { Classical } \\
\text { cold }\end{array}$} & 43 & $t$ & & & 0.002 & 0.000 & 0.000 & 0.038 & 0.000 & & 0.000 \\
\hline & & $f$ & & . & 0.051 & 0.402 & 0.839 & 0.006 & 0.747 & & 0.000 \\
\hline & & KS & & & 0.006 & 0.000 & 0.000 & 0.000 & 0.000 & & 0.000 \\
\hline & & & & & & Not comp. & Not comp. & Not comp. & Not comp. & $-1-$ & Not comp. \\
\hline \multirow{4}{*}{$\begin{array}{l}\text { Classic } \\
\text { hot }\end{array}$} & 28 & $t$ & & & & 0.602 & 0.160 & 0.560 & 0.039 & & 0.000 \\
\hline & & $f$ & & & & 0.377 & 0.106 & 0.471 & 0.094 & & 0.000 \\
\hline & & $\mathrm{KS}$ & & & & 0.161 & 0.002 & 0.284 & 0.040 & & 0.000 \\
\hline & & & & & & Indist. & Marginal & Indist. & Marginal & $-1-$ & Not comp. \\
\hline \multirow{4}{*}{$\begin{array}{l}\text { Scattered } \\
\text { disk } \\
\text { objects }\end{array}$} & 23 & $\bar{t}$ & & & & & 0.355 & 0.286 & 0.114 & & 0.001 \\
\hline & & $f$ & & & & . & 0.406 & 0.125 & 0.569 & & 0.000 \\
\hline & & KS & & & & & 0.280 & 0.358 & 0.359 & & 0.000 \\
\hline & & & & & & & Indist. & Indist. & Indist. & $-1-$ & Not comp. \\
\hline \multirow{4}{*}{$\begin{array}{l}\text { Detached } \\
\text { disk } \\
\text { objects }\end{array}$} & 17 & $t$ & & & & & & 0.064 & 0.583 & & 0.020 \\
\hline & & $f$ & & & & & . & 0.031 & 0.662 & & 0.000 \\
\hline & & KS & & & & & & 0.182 & 0.444 & & 0.000 \\
\hline & & & & & & & & Indist. & Indist. & $-1-$ & Not comp. \\
\hline \multirow{4}{*}{ Centaurs } & 26 & $t$ & & & & & & & 0.015 & & 0.000 \\
\hline & & $f$ & & & & & & & 0.014 & & 0.000 \\
\hline & & KS & & & & & & & 0.035 & & 0.000 \\
\hline & & & & & & & & & Marginal & $-1-$ & Not comp. \\
\hline \multirow{4}{*}{$\begin{array}{l}\text { Short } \\
\text { period } \\
\text { comets }\end{array}$} & 44 & $t$ & & & & & & & & & 0.090 \\
\hline & & $f$ & & & & & & . & & & 0.000 \\
\hline & & KS & & & & & & & & & 0.000 \\
\hline & & & & & & & & & & $-1-$ & Not comp. \\
\hline
\end{tabular}

Notes. The three tests are the Student $t$ - and $f$-tests and the KS test. The number $\mathrm{N}$ indicates how many objects have measurements. The result is the probability that the two distributions are randomly extracted from the same population. The label indicates whether the populations are significantly different (i.e., not compatible), marginally different, or statistically indistinguishable (see text for details).

randomly extracted from a same pool, even though this may be just a coincidence.

Table 5 summarizes the results of the comparison between the spectral gradient $\mathcal{S}$ distribution of the MBOSS populations using these tests. Following the discussion in Sect. 3.2, the tests were restricted to the objects with $M(1,1)>5 \mathrm{mag}$. For each pair, the table lists the probabilities that the two classes were randomly extracted from a common population, based on the mean spectral gradient ( $t$-test), the spectral gradient variance ( $f$-test), and the overall spectral gradient distribution (KS test). Low probabilities indicate that the two considered distributions are incompatible. The pairs for which at least one of the tests returns a probability $<0.001$ were flagged as "not compatible", indicating they are significantly different. Those where a test indicates a probability $<0.05$ are marked as marginally different, suggesting that there is a difference, but that it is not very strongly significant. The others are statistically indistinguishable using these tests, which again does not mean that the objects are similar, just that these tests cannot prove they are different. Similar tests were performed on the individual colours, with similar results. We discuss here only the spectral gradients, as they encompass most of the information present in the visible spectrum range.
In the infrared, the tests are possible only in $J-H$, and only some of the classes have enough measurements for the tests to be meaningful. The results are presented in Table 6. The statistical tests indicate the following results:

- The spectral gradient distributions of the various resonant classes are statistically indistinguishable. To increase the size of the sample, all the resonant objects were divided only between Plutinos (3:2) and "other resonant objects".

- The spectral gradient distributions of Plutinos and other resonant objects, SDOs, hot CDOs, and Centaurs are indistinguishable.

- Additionally, the Plutinos and the hot CDOs had indistinguishable $J-H$ distributions.

- The spectral gradient distribution of DDOs is indistinguishable from that of SDOs and only marginally distinguishable from those of Plutinos and resonant objects, as well as hot CDOs.

- The spectral gradient distribution of cold CDOs is incompatible with the ones of hot CDOs, Plutinos, SDOs, DDOs, and Centaurs, and is only marginally compatible with that of the resonant objects.

- Additionally, the cold CDOs $J-H$ distribution is incompatible with that of the Plutinos and the hot CDOs. 
Table 6. Statistical comparisons of the $J-H$ colour distributions of pairs of MBOSS populations: $t$-test, $f$-test, and KS test.

\begin{tabular}{lccccc}
\hline \hline & & & $\begin{array}{c}\text { Classic } \\
\text { cold }\end{array}$ & $\begin{array}{c}\text { Classic } \\
\text { hot }\end{array}$ & Centaurs \\
\hline & $N$ & & 28 & 34 & 17 \\
\hline Resonant & 21 & $t$ & 0.498 & 0.119 & 0.308 \\
$3: 2$ & & $f$ & 0.000 & 0.961 & 0.000 \\
& & $\mathrm{KS}$ & 0.449 & 0.677 & 0.984 \\
& & & Not comp. & Indist. & Not comp. \\
\hline Classic & 28 & $t$ & & 0.121 & 0.332 \\
cold & & $f$ & - & 0.000 & 0.703 \\
& & $\mathrm{KS}$ & & 0.710 & 0.284 \\
& & & & Not comp. & Indist. \\
\hline Classic & 34 & $t$ & & & 0.271 \\
hot & & $f$ & - & - & 0.000 \\
& & $\mathrm{KS}$ & & & 0.499 \\
& & & & Not comp. \\
\hline
\end{tabular}

Notes. The three tests are the Student $t$ - and $f$-tests and the KS test. The $\mathrm{N}$ number indicates how many objects have measurements. The classes that are not listed do not have sufficient measurements for these tests to be performed. The result is the probability that the two distributions are randomly extracted from the same population. The label indicates whether the populations are significantly different, marginally different, or statistically indistinguishable (see text for details).

- The spectral gradient distribution of Jupiter Trojans clearly indicates its complete incompatibility with all other MBOSS populations.

- The spectral gradient distribution of SPCs significantly differs from those of the Jupiter Trojans and cold CDOs. It is only marginally different from those of the hot CDOs, Plutinos, and other resonant objects as well as Centaurs, and it cannot be distinguished from that of SDOs and DDOs.

- The $J-H$ colours of the Centaurs are incompatible with those of the Plutinos and the hot CDOs.

In conclusion, Plutinos, other resonant objects, hot CDOs, SDOs, and DDOs may have rather similar spectral gradients in the visible, while cold CDOs and Jupiter Trojans form two separate groups of spectral properties in the visible wavelength range. The peak in the spectral gradient distribution of hot CDOs seems to be shifted slightly to higher reddening, although for the moment this result is not yet at a statistically significant level. Short-period comets may agree with the first populations mentioned above.

\subsubsection{Absolute magnitude}

The absolute magnitude $M(1,1)$ of an object depends on the reflectivity function across the surface, i.e. a first order approximation on the object size, the geometric albedo, and the photometric phase function. Given the relationship between $M(1,1)$, size, albedo, and phase function, one can assume that the $M(1,1)$ distribution is dominated by the effects of the size, except for the largest bodies, which often have high albedos (Stansberry et al. 2008). The solar phase angle for KBOs is always relatively small, and the phase function is moderately steep, so the effect on $M(1,1)$ hardly exceeds $0.2-0.3 \mathrm{mag}$. Hence, we assume that the $M(1,1)$ distribution of KBOs reflects - at least to zeroth order - the size distribution of the objects, with the understatement that a detailed analysis requires more accurate size estimates of the objects. Such a careful study can be found in Stansberry et al. (2008).
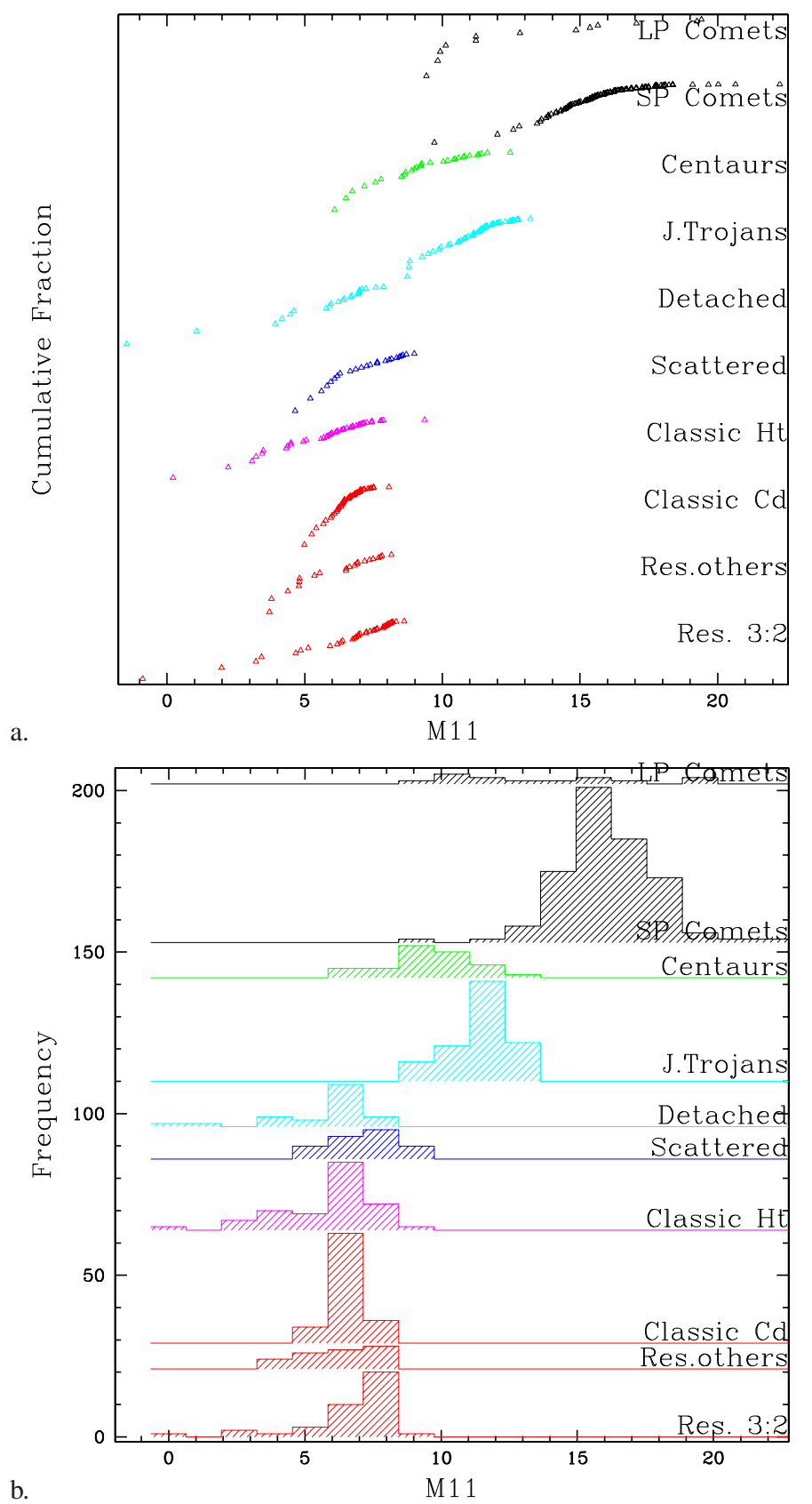

Fig. 6. $R$ absolute mag nitude $M(1,1)$ cumulative distributions (logarithmic; a), and histogram $\mathbf{b})$.

The $M(1,1)$ distributions are strongly biased by cumulative selection effects: while there are few bright and large objects in a given class, they are more easy to discover, and also more easy to observe, so they are likely to be represented in this data-set. A contrario, the discovery surveys are incomplete for fainter objects, and faint objects are less likely to be picked by observers performing colour measurements. Consequently, the $M(1,1)$ distributions are likely to be fairly complete at the bright end, but have a complex non-completeness at the faint end. Meech et al. (2004) discussed and simulated many of these selection effects.

Figure 6 shows the frequency histogram and cumulative distribution function of $M(1,1)$ for the various MBOSS groups, now covering all objects in the MBOSS database (i.e. not excluding those with $M(1,1)>5 \mathrm{mag})$. 
O. R. Hainaut et al.: Colours of minor bodies in the outer solar system. II.

The selection bias against small KBOs is clear, with no object fainter than $M(1,1) \sim 8.5$ in the distant classes - this corresponds to actual magnitudes fainter than 23.5 for Plutinos, and even fainter for CDOs, i.e. about the limiting magnitude for colour observations performed with fairly short exposures on a $8 \mathrm{~m}$ class telescope. On the other hand, Centaurs and SPCs may be seen as a sample of smaller KBOs that is scattered towards the inner planetary system. Meech et al. (2004) found that the shape of the SPC size distribution is incompatible with that of the other TNOs.

The slope $k$ of the logarithmic cumulative $M(1,1)$ distribution relates to the power law $q$ of the cumulative radius distribution $N_{\mathrm{c}}(a) \propto a^{-q}$ as $q=5 \mathrm{k}$. Measuring the $k$ slopes on the straight parts of the distributions in Fig. 6 leads to values $q \sim 0.8$ for the SPCs, the Centaurs, the faint SDOs, the DDOs, the hot CDOs, and the resonant objects; the steep slope of the cold CDOs and the bright SDOs corresponds to $q \sim 2$. Only the steepest part of the SPCs distribution reaches $q \sim 3$. Analyses based on controlled samples designed for size analyses have found much steeper power-laws of from $q=3.0$ (Trujillo et al. 2001 ) to 3.5 (Kenyon \& Bromley 2004), i.e. distributions much richer in small/faint objects. This confirms that the sample used for colour measurements is heavily biased against faint objects the distributions may be fairly complete only for the brightest SPCs.

Therefore, the size distributions in Fig. 6 should only be used for internal comparison, with the hope that the selection effects affected them in a similar way, and/or restricting the analysis to the bright end of the distributions.

The observed Jupiter Trojans $(M(1,1)$ between 8 and $13 \mathrm{mag}$ ) are clearly smaller than KBOs, which cover the $M(1,1)$ range from about 0 to $9 \mathrm{mag}$. The bulk of that shift is caused by the proximity of the Trojans, which enables objects fainter by $\sim 4$ mag to be observed.

It is noteworthy that cold CDOs display a rather peaked $M(1,1)$ distribution and do not contain objects with $M(1,1)$ brighter than about $5 \mathrm{mag}$, while Plutinos, hot CDOs, and DDOs contain objects of $M(1,1)$ from 2 to -1 mag. The rather steep slope in the cumulative distribution function of $M(1,1)$ of the cold CDOs reflects the likely difference in the size range of these KBOs. In particular, it differs from that of hot CDOs, which should be exposed to very similar selection effects, i.e. it is real. The brightest resonant objects reach $M(1,1)$ of about 3 mag, while the SDOs are more similar in brightness to cold CDOs, despite having a much less pronounced peak level on a slightly wider distribution plateau.

From the $M(1,1)$ distributions of MBOSSes, we conclude that the absence of larger bodies among the cold CDOs and their presence among other populations of the Kuiper belt may suggest different formation environments for both groups of objects that may have favored the growth of larger bodies for Plutinos, SDOs, DDOs, and possibly hot CDOs compared to cold CDOs. Jupiter Trojans are a population of smaller size bodies, again arguing for a formation environment and evolution scenario that were distinct from that of the other MBOSSes (now including cold CDOs). Since it is very likely that the large bodies in these groups may have survived impact events widely unaffected over the lifetime of the solar system, one can assume that they represent the original population of planetesimals. The Centaurs reach the magnitude/size range where the vast majority of larger KBOs are detected, but they do not include one of the rare big ones. This might well be compatible with the scattering scenario for Centaurs but needs quantitive confirmation by dynamical calculations.

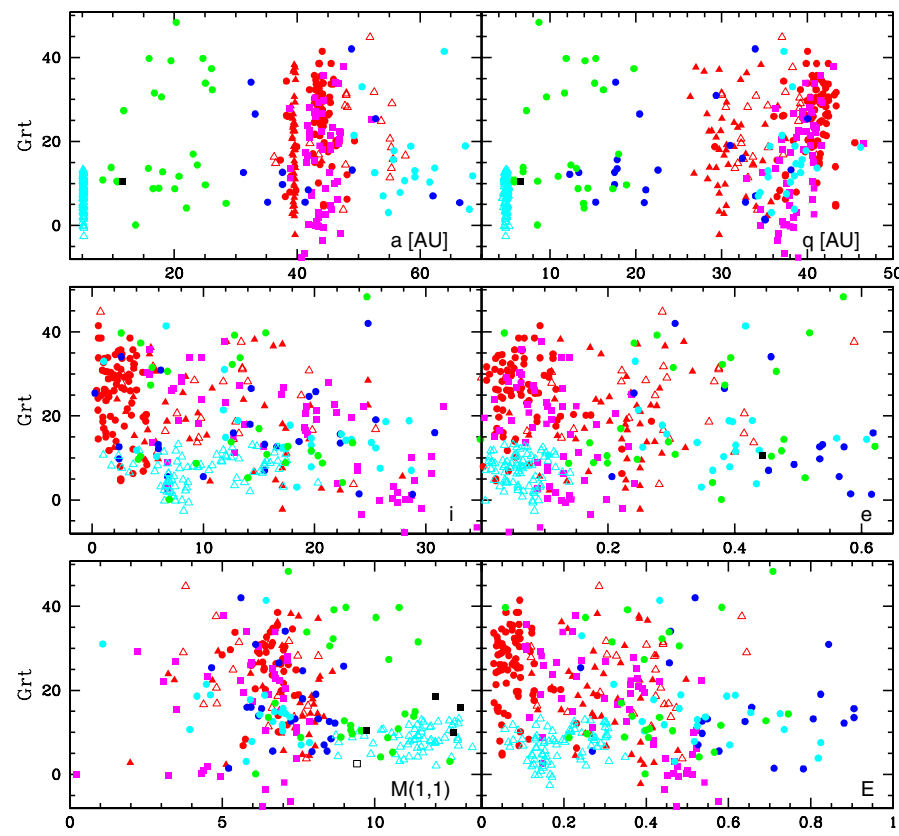

Fig. 7. The spectral gradient of the objects $[\% / 100 \mathrm{~nm}]$ as a function of the main orbital elements, the orbital excitation $\mathcal{E}$, and the absolute $R$ magnitude $M(1,1)$. Similar plots are available on-line for the various colours.

Altogether, the $M(1,1)$ distributions give the impression that the MBOSSes may have been formed in at least three (maybe four) different environments, i.e. one for the Jupiter Trojans, one for the cold CDOs, maybe one for the hot CDOs, and one for the rest of the bodies. Since KBOs now reside and for a very long time resided in the same distance range, one may consider Plutinos, resonant objects, hot CDOs, and SDOs as immigrant population of the Kuiper belt compared to the cold CDOs that may represent "aborigines KBOs". Interestingly, the DDOs also appear to be "sizewise" in accord with the Kuiper belt "immigrants", although they are found at a much more distant location.

\subsection{Dependences on dynamical parameters}

The colour distribution (say, the $B-V$ distribution) of a class of objects (say, the Centaurs) is considered as a function of another parameter of the objects (say, the perihelion distance $q$ ) to search for correlations as possible indicators of any physical dependences. The traditional Pearson correlation factor is not robust for non-linear dependencies, so a simpler and more robust estimator was used: the sample is divided into two sub-samples, using the median value of the independent variable (in the example, the median $q$ of the Centaurs, i.e. 16.22 AU). The colour distribution of the two sub-samples are then compared through $t$-test and $f$-test and the Pearson correlation parameter is also estimated. Visually, this can be done by comparing the left-hand side with the right-hand side of all the sub-panels of Fig. 7.

For cold CDOs: the variances in the colours $B-V, B-R$ and $B-I$ are different for the inner (smaller variance) and the outer (larger variance) population among the cold CDOs (considering both their semi-major axis and perihelion distance). These differences have a good level of significance, as listed in Table 8. The effect is only marginally observed in $V-R$ and $V-I$. The average colours of the sub-samples are indistinguishable: only the breaths of the distributions are different. 


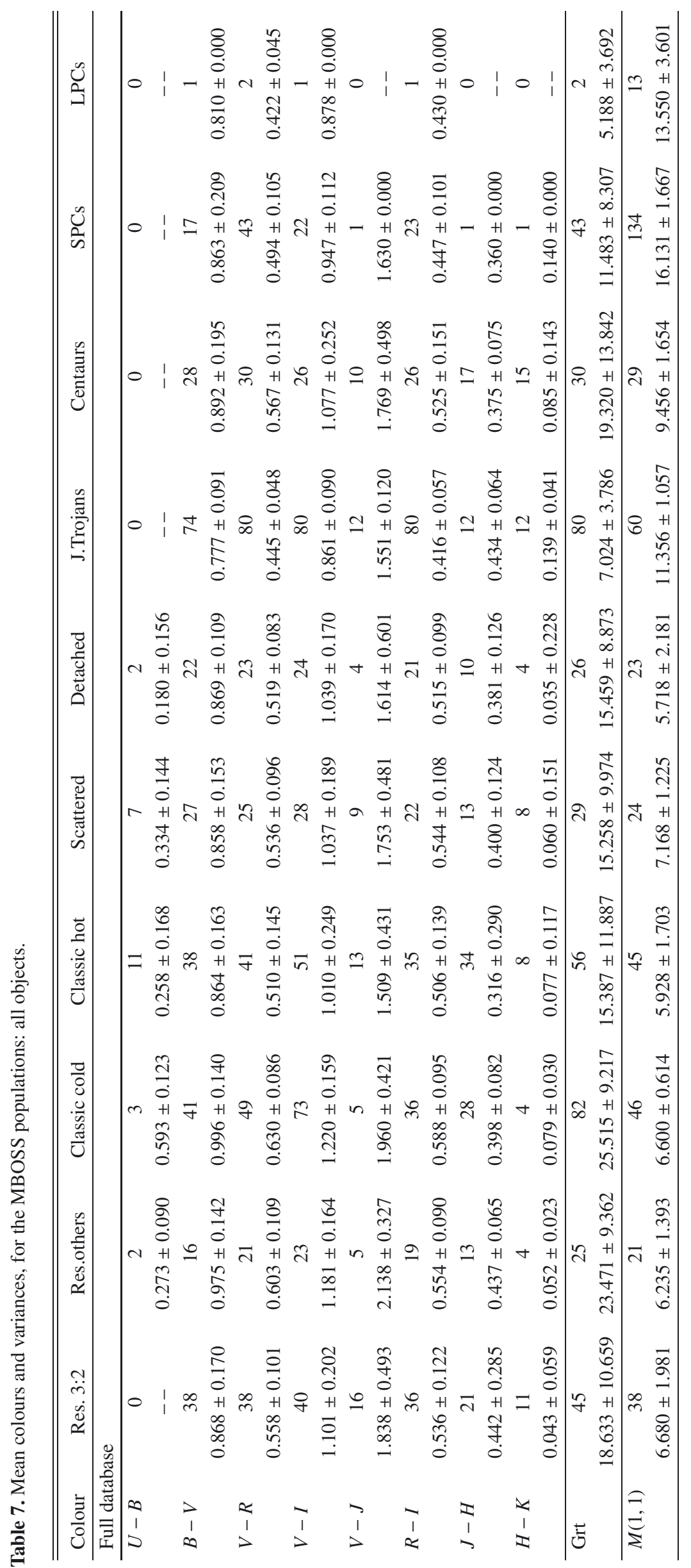


O. R. Hainaut et al.: Colours of minor bodies in the outer solar system. II.

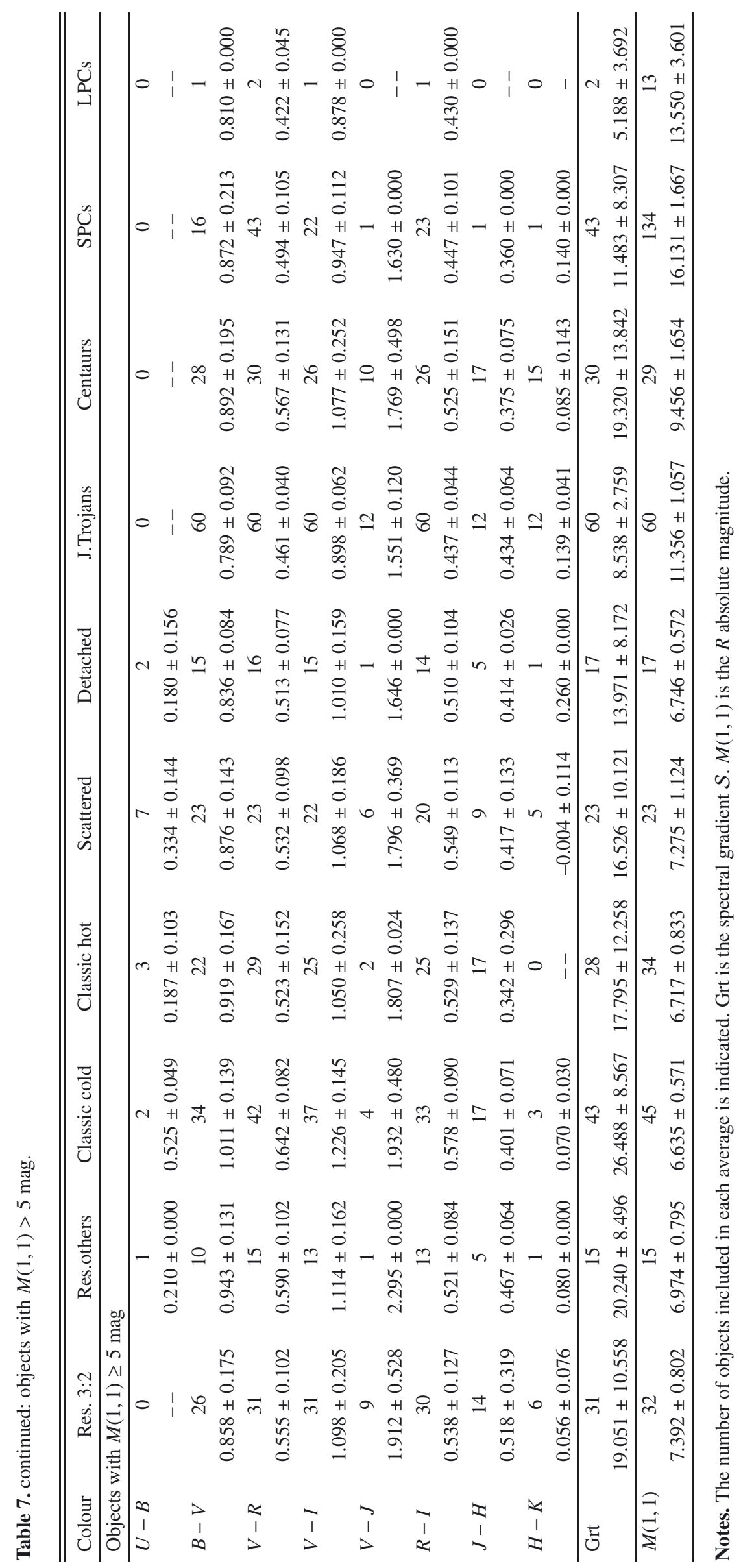


Table 8. Averge colours and dispersions for the cold CDOs, comparing those with smaller $a$ (or $q$ ) and those with large values.

\begin{tabular}{|c|c|c|c|c|c|c|}
\hline Colour & $N$ & $\begin{array}{r}\quad \text { Aver. } / \sigma \\
a<43.95\end{array}$ & $N$ & $\begin{array}{l}\quad \text { Aver./ } \sigma \\
a \leq 43.95\end{array}$ & $t$-Prob & $f$-Prob \\
\hline$B-V$ & 16 & $0.99 \pm 0.16$ & 18 & $1.03 \pm 0.11$ & 0.148 & 0.148 \\
\hline$B-R$ & 16 & $1.59 \pm 0.26$ & 17 & $1.69 \pm 0.09$ & 0.151 & 0.000 \\
\hline$B-I$ & 9 & $2.18 \pm 0.37$ & 14 & $2.27 \pm 0.15$ & 0.489 & 0.003 \\
\hline$V-R$ & 21 & $0.63 \pm 0.10$ & 21 & $0.65 \pm 0.06$ & 0.310 & 0.034 \\
\hline$V-I$ & 17 & $1.20 \pm 0.16$ & 20 & $1.24 \pm 0.14$ & 0.395 & 0.538 \\
\hline & \multicolumn{2}{|r|}{$q<40.91$} & \multicolumn{2}{|r|}{$q \leq 40.91$} & & \\
\hline$B-V$ & 17 & $0.95 \pm 0.17$ & 17 & $1.07 \pm 0.06$ & 0.013 & 0.000 \\
\hline$B-R$ & 16 & $1.56 \pm 0.25$ & 17 & $1.72 \pm 0.07$ & 0.024 & 0.000 \\
\hline$B-I$ & 13 & $2.15 \pm 0.30$ & 10 & $2.34 \pm 0.13$ & 0.060 & 0.017 \\
\hline$V-R$ & 19 & $0.61 \pm 0.08$ & 23 & $0.67 \pm 0.08$ & 0.033 & 0.802 \\
\hline$V-I$ & 18 & $1.20 \pm 0.18$ & 19 & $1.25 \pm 0.10$ & 0.278 & 0.010 \\
\hline
\end{tabular}

Notes. The $a$ and $q$ cut-off are set at the median value; $N$ is the number of measurements; $t$-Prob and $f$-Prob are the probabilities that the two sub-samples are randomly extracted from the same distribution, evaluated with the $t$ - and $f$-tests. The other colours show insignificant differences.

Table 9. Average colours and dispersions for the hot CDO, comparing those with smaller $q$ and those with large values.

\begin{tabular}{lcccccc}
\hline \hline Colour & $N$ & $\begin{array}{c}\text { Aver. } / \sigma \\
q<39.39\end{array}$ & $N$ & $\begin{array}{c}\text { Aver. } / \sigma \\
q \leq 39.39\end{array}$ & $t$-Prob & $f$-Prob \\
\hline$B-V$ & 10 & $0.80 \pm 0.12$ & 12 & $1.02 \pm 0.13$ & 0.000 & 0.795 \\
$B-R$ & 10 & $1.28 \pm 0.21$ & 12 & $1.64 \pm 0.18$ & 0.000 & 0.666 \\
$B-I$ & 8 & $1.71 \pm 0.28$ & 9 & $2.37 \pm 0.27$ & 0.001 & 0.838 \\
$V-R$ & 14 & $0.47 \pm 0.14$ & 15 & $0.57 \pm 0.15$ & 0.071 & 0.792 \\
$V-I$ & 12 & $0.94 \pm 0.24$ & 13 & $1.15 \pm 0.24$ & 0.037 & 0.967 \\
\hline
\end{tabular}

Notes. The $q$ cut-off is set at the median value; $N$ is the number of measurements; $t$-Prob and $f$-Prob are the probabilities that the two subsamples are randomly extracted from the same distribution, evaluated with the $t$ - and $f$-tests. The other colours show insignificant differences.

Since similar results are found neither in other colours nor the spectral gradients in the visible, the effect might be related to a wavelength dependent phenomenon that is mostly restricted to the B filter, for instance a stronger unknown continuum absorber in the outer cold CDOs.

For hot CDOs: hot CDOs with a perihelion closer to the Sun are significantly bluer than the ones that do not get close, see Table 9, confirming earlier suggestions by Boehnhardt et al. (2004) based on a smaller object sample. This result is visible in the $B-V, B-R$, and $B-I$ colours (e.g. $B-V=0.80 \pm 0.12$ for $q<39.4 \mathrm{AU}$, and $B-V=1.02 \pm 0.13 \mathrm{mag}$ for the others). This result is based on fairly small samples, which decreases the strength of its significance.

Hot CDOs with high inclinations or with large orbital excitation parameters are bluer than the others, see Table 10, a finding seen for several colours $(B-V, B-R, V-R, V-I)$ as well as for the spectral gradient in the visible. The sample sizes are small, but sufficient. This result was already known (Trujillo \& Brown 2002; Doressoundiram et al. 2008) and is generally expressed as an anti-correlation between reddening of the objects and their orbital energy $\mathcal{E}$. The usual interpretation scenario is colour changes due to resurfacing by impact ejecta if during a collision between a hot $\mathrm{CDO}$ and another KBO subsurface material with different colour properties is ejected and deposited on the surface by impact excavations. Since cold CDOs do not
Table 10. Average colours and dispersions for the hot CDOs, comparing those with smaller $i$ and those with large values.

\begin{tabular}{|c|c|c|c|c|c|c|}
\hline Colour & $N$ & $\begin{array}{c}\quad \text { Aver. } / \sigma \\
i<17.0\end{array}$ & $N$ & $\begin{array}{c}\quad \text { Aver./ } \sigma \\
i \leq 17.0 \\
\end{array}$ & $t$-Prob & $f$-Prob \\
\hline & 14 & $090+016$ & 8 & $080+000$ & & \\
\hline & 14 & & 8 & & & \\
\hline$B-I$ & 11 & & 6 & & & \\
\hline$V-R$ & 15 & 0.61 & 14 & 0.43 & & 0.012 \\
\hline$V-I$ & 13 & $1.20 \pm 0.15$ & 12 & $0.89 \pm 0$ & 0.002 & 0.064 \\
\hline Grad. & 15 & $24.59 \pm 7.99$ & 13 & $9.95 \pm 11.80$ & 0.001 & 0.165 \\
\hline
\end{tabular}

Notes. The $q$ cut-off is set at the median value; $N$ is the number of measurements; $t$-Prob and $f$-Prob are the probabilities that the two subsamples are randomly extracted from the same distribution, evaluated with the $t$ - and $f$-tests. The other colours show insignificant differences.

display such an anti-correlation despite of their occupying the same distance range as the hot CDOs do, it may be more an effect of orbital energy, i.e. collision energy rather than collision frequency.

For others: other combinations of dynamical parameters and colours, spectral gradient, and $M(1,1)$ data of different dynamical classes do not show any statistically significant signal indicating possible correlations and physical dependencies.

\subsection{Absolute magnitude}

\subsubsection{Absolute magnitude vs. orbital parametres}

For the comparison between $M(1,1)$ and dynamical parameters, we consider the small objects $(M(1,1) \geq 5 \mathrm{mag})$ only and we find that, for Centaurs, SDOs, and DDOs, when considered together, objects with small semi-major axis $a$ and perihelion distance $q$ display a significantly fainter absolute magnitude $M(1,1)$ than the distant ones. While this is a statistically strong result, it disappears when the three classes are considered separately. Thus, it can simply be seen as the selection bias that makes the more distant and thus fainter objects harder to measure than the closer ones.

\subsubsection{Absolute magnitude of the blue objects, vs. red objects:}

We now compare the absolute magnitude $M(1,1)$ distribution of a class of objects as a function of their colour, to explore whether the redder objects have the same $M(1,1)$ distribution as the bluer objects. Considering only the small objects $(M(1,1) \geq 5 \mathrm{mag})$, the blue hot CDOs and the blue resonant objects have fainter absolute magnitudes than the redder objects of the same type (see Table 11).

\subsubsection{Others parameters}

Other combinations of the absolute magnitude with other parameters do not show any statistically significant signal.

\section{Interpretation scenarios}

In the following discussion, we try to elaborate and and understand the results from the statistical analysis described in Sect. 3 in the framework of two rather different scenarios, i.e. surface colouration by on-going external processes and surface colours as a result of the primordial constitution of the bodies. 
Table 11. Average $M(1,1)$ and dispersions for the hot CDOs, comparing those with blue vs. red colours.

\begin{tabular}{lcccccc}
\hline \hline Colour & $\begin{array}{c}\text { Colour } \\
\text { cut-off }\end{array}$ & $N$ & $\begin{array}{c}\text { Aver. } / \sigma \\
\text { Blue }\end{array}$ & $N$ & $\begin{array}{c}\text { Aver. } / \sigma \\
\text { Red }\end{array}$ & $t$-Prob \\
\hline Hot TNOsO & & & & & & \\
$B-V$ & 0.92 & 11 & $6.92 \pm 0.67$ & 11 & $6.54 \pm 0.62$ & 0.183 \\
$B-R$ & 1.55 & 11 & $7.09 \pm 0.45$ & 11 & $6.37 \pm 0.64$ & 0.007 \\
$B-I$ & 1.92 & 8 & $7.13 \pm 0.52$ & 9 & $6.41 \pm 0.67$ & 0.025 \\
$V-R$ & 0.56 & 14 & $6.88 \pm 0.61$ & 15 & $6.34 \pm 0.62$ & 0.026 \\
$V-I$ & 1.10 & 12 & $6.87 \pm 0.61$ & 13 & $6.29 \pm 0.65$ & 0.031 \\
$R-I$ & 0.54 & 12 & $6.85 \pm 0.53$ & 13 & $6.30 \pm 0.72$ & 0.039 \\
\hline Resonant & objects & & & & & \\
$B-V$ & 0.88 & 18 & $7.61 \pm 0.81$ & 18 & $6.79 \pm 0.78$ & 0.004 \\
$B-R$ & 1.40 & 18 & $7.61 \pm 0.81$ & 18 & $6.79 \pm 0.78$ & 0.004 \\
$B-I$ & 1.89 & 15 & $7.40 \pm 0.84$ & 16 & $6.79 \pm 0.81$ & 0.052 \\
$V-R$ & 0.56 & 23 & $7.48 \pm 0.72$ & 23 & $7.04 \pm 0.88$ & 0.073 \\
$V-I$ & 1.06 & 22 & $7.42 \pm 0.72$ & 22 & $7.08 \pm 0.91$ & 0.170 \\
$R-I$ & 0.51 & 21 & $7.47 \pm 0.78$ & 22 & $7.01 \pm 0.83$ & 0.065 \\
\hline
\end{tabular}

Notes. The objects with $M(1,1)<5$ were excluded from this test; the colour cut-off is set at the median value for the considered colour; $N$ is the number of measurements; $t$-Prob are the probabilities that the two sub-samples are randomly extracted from the same distibution, evaluated with the $t$-test. The other colours show insignificant differences.

\subsection{Surface colouration by external processes}

Colour diversity of MBOSSes was originally attributed to the action of external processes on their surfaces. These process included resurfacing by impacts, which were expected to produce the neutral-bluish colours of fresh ice excavated and/or spread over the surface, and an irradiation process, producing red colours via radiation aging (e.g. Luu \& Jewitt 1996, for a model). When restricting our MBOSS database samples to objects with $M(1,1) \geq 5 \mathrm{mag}$, as done during most of our analysis, a third process, i.e. resurfacing due to atmospheric deposits of material from intrinsic activity, was considered less important for the subsequent discussion, since it should only affect largesized bodies, the smaller ones losing the material into space.

A critical argument for significant changes in the surface reflectivity due to impacts comes from a lightcurve analysis of MBOSSes: asymmetric or partial impact resurfacing of the body surfaces should cause colour variegations over the rotation of the bodies that are, however, usually not seen (Sekiguchi et al. 2002; Sonnett, priv. comm.; Ortiz, priv. comm.). Nevertheless, other authors report colour changes with rotation phase, e.g. for Haumea (Lacerda et al. 2008). Thus, strong variegation is certainly not widespread.

In the colouration scenario based on external processes, DDOs are expected to represent the end state of the radiation reddening of the surface, since one can assume that, for these objects, impact resurfacing does not occurs at a significant level, simply because the spatial number density of DDOs seems very low and collisions should therefore happen extremely rarely over the lifetime of the solar system. From our statistical analysis, we found that DDOs are not the reddest objects among MBOSSes. Cold CDOs instead show by far the reddest surface colours. To explain the smaller reddening of DDOs in this colouring scenario, one has to assume that cold CDOs do not represent the end state of radiation reddening (despite showing the reddest colours), but high radiation doses, similar to those to which DDOs are exposed, decrease the spectral gradients again. Laboratory experiments (de Bergh et al. 2008) have found materials that display such behaviour, although the end state of these material has rather neutral spectral gradients in the visible wavelength range. However, it remains unclear whether these materials exist in DDOs and, if not, whether the laboratory results apply more generally to more or even all possible surface compounds considered to exist in DDOs. It is also noteworthy to mention the coincidence of the peaks in the spectral gradient distributions of DDOs as well as SDOs, Plutinos, resonant objects, and Centaurs; this may be by chance despite the different importance of collisional resurfacing for these groups.

The colour cascade, with peaks in the colour histograms decreasing in reddening from cold CDOs to hot CDOs, then SDOs, Plutinos, resonant objects, and parts of the Centaurs, must be attributable to the increasing importance of impact resurfacing. However, Centaurs live in a rather empty zone of the planetary system, hence impact resurfacing must contribute less to their evolution. Here, the peak in the spectral gradient diagram could be attributed to resurfacing from intrinsic activity. The very red Centaurs instead represent the inactive objects that are subject to only radiation reddening, though not with extremely high doses (otherwise they could be expected to show similar reddening to DDOs). Among the six known Centaurs showing cometary activity, currently only one has red colours. A larger sample of colours of cometary active Centaurs is therefore needed.

Jupiter Trojans differ in terms of their surface reddening from the MBOSSes. It is remarkable that Jupiter Trojans do not show very "red" surfaces as objects in all other MBOSS populations do, despite their high exposure to radiation from the Sun (owing to their closer distance by a factor of about 100). Thus, we conclude that this difference may be due to their having different material properties from the other MBOSSes.

An interesting result is seen for the SPCs: the population's peak is well within the range of those of SDOs, Plutinos, moderately red Centaurs, as well as DDOs. Impact resurfacing should be negligible for SPCs and instead resurfacing due to activity should be far more important. On the other hand, owing to the fast, continuous mass-loss on short time-scales (the lifetime for active SPCs is estimated to be 100000 years or less), the surface deposits should represent the intrinsic colour of the cometary material. Radiation aging is unimportant over the lifetime of SPC nuclei in the inner planetary system since cometary activity will result in fast and continuous resurfacing of the body. Hence, SPCs give the impression that their nuclei may consist on a global scale - of material with rather homogeneous colour properties. As SPCs to a large extent originate from the Kuiper belt (Gomes et al. 2008; Morbidelli et al. 2008a) and because of their colour similarity to the immediate dynamical relatives therein (SDOs, Plutinos) and the Centaurs from where SPCs are captured, it is attractive to assume that the colours of these relatives should also be globally uniform throughout the bodies. Jewitt (2002) discusses the missing very red SPCs, suggesting that the red surfaces are rapidly buried as water ice sublimation starts at $r<6 \mathrm{AU}$.

\subsection{Global colouration in the formation era}

An alternative interpretation of the global results for the colour and $M(1,1)$ distribution is that the measured colours actually reflect the global characteristics of the objects, that is throughout the object and not only to its (aged) surface. This hypothesis is supported by the interpretation of SPC colours and the similarities of the peaks in the colour distribution of the MBOSS populations. We note that Brown et al. (2011) introduced a similar hypothesis for the colour diversity among Kuiper belt objects 
based on sublimation properties of super-volatile ices. We describe below what can be seen as arguments in support of such a "primordial" colouration scheme for the MBOSSes, where "primordial" indicates that the colours of the body materials originate from a time before the body was formed, i.e. the formation period of the planetary system, and have remained largely unaltered ever since.

An additional assumption needed is that the surface materials are not - or at least not by a large amount - affected by external processes such as reddening due to cosmic radiation. The global character of the material colours also implies that impact resurfacing would reproduce the original pre-impact surface colours of the bodies and that large-scale colour diversities on the surface should not occur. Intrinsic activity in MBOSSes will expel gaseous species and solids of which at least the solids may resemble the original colours, while the gases, if not lost to interplanetary space, may condense on the body as surface frost, in which case the surface colour may change. However, our selection criterion to use only objects with $M(1,1)>5$ mag for the statistical analysis should significantly reduce the possibility that MBOSSes that may display intrinsic activity "contaminate" the study results.

In the context of this primordial colouration scenario, the findings from our statistical analysis of the MBOSS colours allow us to draw some interesting conclusions:

- At least four different colour populations may have existed in the protoplanetary disk, i.e. the cold CDOs, the hot CDOs, the SDO/DDO/Plutino populations, and the Jupiter Trojans. The four populations are characterized by different surface colours and spectral gradients of decreasing amplitude in the visible wavelength region, but may have very similar colours in the NIR.

- The colour diversities among MBOSS populations with different surface colours call for formation environments at different and disjunct distances from the Sun. Migration transport may have shifted the original populations to their current locations in the planetary system, that is have placed cold and hot CDOs in the same distance range. This is very much in line with the Nice model - see Sect. 7.1 of Morbidelli et al. (2008a) for an overall description, or Levison et al. (2008) for more details, which gives an integrated overview of the dynamical history of the outer solar system. In the Nice model, the cold CDOs form a distinct population formed at larger distances from the Sun than the other classes, and in which the hot CDOs are then implanted in the later stages of Neptune's outward migration. The Nice model also specifically accounts for the separate nature of the Jupiter Trojans (Morbidelli et al. 2005) and is able to produce DDOs from a population at closer distances to the Sun than the cold CDOs. The Nice model lists an impressive list of successes in reproducing the dynamical structure of the current solar system; the observed similarity of the SDO, DDO, and resonant populations could help refining the overall picture painted by the model. However, DDOs and hot CDOs may represent a challenge for migration scenarios, in particular if one wants to include their colour similarities with other MBOSS populations.

- Similar colour properties among MBOSS populations at different locations in the outer planetary system may indicate a common formation region of the bodies. If so, this could apply to both Plutinos and DDOs, objects nowadays found at rather different distances from the Sun. Migration (for the Plutinos) and scattering (for the DDOs) might again have been involved in separating the bodies formed in a common source region.

- The migration and scattering processes have kept a population of the same formation origin together and shifted them to confined destination regions with no obvious signs of intermixing from other sides. This conclusion suggests that the migration and scattering processes in the outer solar system might not have been very violent since they have retained the colour characteristics as population entities.

- The colour distributions for the populations with "temporary" dynamical object association, i.e. SDOs, Centaurs, and SPCs, should reflect the efficiency of the injection and ejection processes of bodies from the various feeding populations, i.e. Plutinos and both hot and cold CDOs.

There are supportive and also critical arguments from the statistical analysis for the conclusions above. In particular, the similarities and differences in the $M(1,1)$ distributions provide compatible pieces of evidence: the disjunct $M(1,1)$ distributions of the Jupiter Trojans and both the hot and cold CDOs (the latter having been noted in various papers and summarized in Doressoundiram et al. 2008) may call for different formation environments at different solar distances, as also suggested by Morbidelli et al. (2008b). On the other side, the existence of large objects with $M(1,1)<3$ mag among Plutinos and DDOs constitutes an independent supportive argument for a formation region that would be common among these populations and distinct from the cold CDOs, as noted by Levison \& Stern (2001) and developed by Gomes (2003). Despite the possibly different formation environments of Plutinos and DDOs on the one hand and hot CDOs on the other as suggested by their colour differences, the presence of large objects $(M(1,1)<3$ mag) in all three populations suggests that the planetesimal formation and destruction processes were similarly efficient. On the other side, no obvious explanation - except an ad hoc assumption as an outcome of the formation - can be offered for the anticorrelation between surface colours and excitation parameter as well as the inclinations of the hot CDO population, since the migration process should be "colour-blind". While the existence of very red objects among hot CDOs, DDOs, and Plutinos could be attributed to the contamination of cold CDOs, the presence of bodies with blue, neutral surfaces in all populations except for cold CDOs and DDOs may indicate that there are colour contaminants either from the primordial environment or due to dynamical injection during a later phase.

\section{A summarizing discussion}

The MBOSS database first published in 2003 has since been updated using subsequently published magnitude and colour measurements of TNOs, Centaurs, Jupiter Trojans, and both shortand long-period comets. A qualifying criterion has been applied to the data in the database in order to distinguish noisy measurement sets from more consistent ones. we have performed a new statistical analysis of the qualified colours, spectral gradient, and absolute $R$ magnitudes $M(1,1)$ data in the database with the aim of identifying group properties among the various dynamical MBOSS populations, i.e. for the hot and cold classical disk objects (CDOs), the scattered disk objects (SDOs), the Plutinos, and resonant objects, as well as related objects outside the Kuiper belt such as detached disk objects (DDOs), Centaurs, and SPCs, using a classification based on the SSBN08 system Gladman et al. (2008). Furthermore, Jupiter Trojans are 
O. R. Hainaut et al.: Colours of minor bodies in the outer solar system. II.

included in the analysis since, from all that is known, these objects are unrelated to the MBOSSes, although represent an abundant population at the border of this region in the solar system. To avoid or at least to reduce the impact on the surface colours due to intrinsic activity, the statistical sample was restricted to objects with normalized (but not yet phase corrected) brightness $M(1,1) \geq 5$ mag.

Our statistical analysis has uncovered indications of indistinguishable and incompatible colour distributions and both the differences and similarities of the $M(1,1)$ parameters among the MBOSS populations. These results are consistent with a scheme of four different colour (and $M(1,1)$ ) groups in the MBOSS regime, i.e. (1) cold CDOs; (2) hot CDOs; (3) Plutinos, resonant objects, SDOs, DDOs, Centaurs, and SPCs; and (4) Jupiter Trojans. Additional correlations between photometric and dynamical parameters of the various populations were searched for, but only a single well-established one was found in the data of the hot CDOs, for which colour anti-correlates with inclination and the collision energy parameter.

The statistical results have been discussed in the context of two basic interpretation scenarios, i.e. by explaining the surface colours of the MBOSSes as being (1) due to the interplay of resurfacing of impact ejecta (blue colour shift) and radiation reddening (red colour shift) and (2) of primordial nature imposed by the material properties of the formation environment. The two scenarios have the principle capability to explain our findings based on the analysis of the MBOSS database. However, they can also be challenged, each of them in a different way. The resurfacing scenario has to work out whether and under which assumption it is able to reproduce the actually observed colour, spectral gradient, and $M(1,1)$ distributions of the MBOSSes and in particular that it can explain the relatively moderate reddening of the DDOs in this picture. In this respect, further lab measurements of the radiation reddening of reference materials under high dose levels could play an important role.

The primordial surface-colour scenario provides a good explanation of the apparent uniformity of surface colours among SPCs despite the cometary activity that should be able to excavate material layers of different colours if they exist throughout the nuclei. The primordial surface-colour scenario works with ad hoc assumptions about the colour, spectral gradient, and $M(1,1)$ parameter distributions that require justification. Brown et al. (2011) indicated some first arguments to this respect. However, beyond that, the scenario interpretation relies on the object migration for the transport of the MBOSSes from their formation environment to the various regions where they are found today. Object migration in the early planetary system has already been introduced to explain the existence of the hot CDOs in the immediate neighborhood of the cold CDOs in the Kuiper belt. More comprehensive models have been introduced (see Morbidelli et al. (2008b) and Gomes et al. (2008) for reviews). These dynamical models are - at the moment - mostly "colour-blind" and "size-blind" apart from an a-priori postulation of colour differences for hot and cold CDOs. Our analysis of the MBOSS colours and $M(1,1)$ size parameters would require at least three, possibly four, different source regions in the primordial disk. It is not very plausible that the MBOSSes remained in these original source regions, but it is instead very likely that they were redistributed after formation to the locations in and out of the Kuiper belt where we find them today. A comprehensive model scenario is not yet available that is able to consistently explain the formation environment, the physical properties and the dynamical history of the MBOSS population. Nonetheless, the dynamics, colour, and size distributions together (possibly also albedos) of the measured populations are to be seen as important and constraining benchmarks for these model scenarios - not only qualitatively, but also quantitatively.

In parallel, considerable progress has been achieved in the taxonomic classification of the objects. This process aims to sort the objects among categories defined by similar colour properties (independently of the physico-dynamical classes - see Barucci et al. 2005a), that is an approach orthogonal to the one presented here, which aims to compare the photometric properties of physico-dynamical classes. The multi-pronged approaches of dynamical simulations and modelling, taxonomic classification, and photometric analysis is moving towards an integrated understanding of the MBOSS populations.

Acknowledgements. H. Boehnhardt wishes to thank the European Southern Observatory for supporting this work during his research stay in September and November 2011 at ESO Headquarters in Garching/Germany. We are grateful for the very valuable comments and input provided by the anonymous referee. This research has made use of NASA's Astrophysics Data System.

\section{References}

Barucci, M. A., \& Fulchignoni, M. 1990, in Asteroids, Comets III (Uppsala universitet Reprocentralen), 7

Barucci, M. A., Doressoundiram, A., Tholen, D., Fulchignoni, M., \& Lazzarin, M. 1999, Icarus, 142, 476

Barucci, M. A., Belskaya, I. N., Fulchignoni, M., \& Birlan, M. 2005a, AJ, 130, 1291

Barucci, M. A., Cruikshank, D. P., Dotto, E., et al. 2005b, A\&A, 439, L1

Barucci, M. A., Brown, M. E., Emery, J. P., \& Merlin, F. 2008, in The Solar System Beyond Neptune, eds. M. A. Barucci, H. Boehnhardt, D. P. Cruikshank, A. Morbidelli, \& R. Dotson (U. Arizona Press), 143

Bauer, J. M., Meech, K. J., Fernández, Y. R., et al. 2003, Icarus, 166, 195

Benecchi, S. D., Noll, K. S., Stephens, D. C., Grundy, W. M., \& Rawlins, J. 2011, Icarus, 213, 693

Boehnhardt, H., Tozzi, G. P., Birkle, K., et al. 2001, A\&A, 378, 653

Boehnhardt, H., Barucci, M. A., \& Delsanti, A., et al. 2004, in The First Decadal review of the Edgeworth-Kuiper belt, eds. J. K. Davies, \& L. J. Barrera (Kluwer Scientific Publishers), 145

Brown, M. E. 2008, in The Solar System Beyond Neptune, eds. M. A. Barucci, H. Boehnhardt, D. P. Cruikshank, A. Morbidelli, \& R. Dotson (U. Arizona Press), 335

Brown, M. E., Trujillo, C. A., \& Rabinowitz, D. L. 2005, ApJ, 635, L97

Brown, M. E., Schaller, E. L., \& Fraser, W. C. 2011, ApJ, 739, L60

Coradini, A. 1977, Comput. Geosci., 3, 85

Davies, J. K. 2000, in Minor Bodies in the Outer Solar System, eds. A. Fitzsimmons, D. Jewitt, \& R. M. West (Springer), 9

Davies, J. K., McBride, N., Ellison, S. L., Green, S. F., \& Ballantyne, D. R. 1998, Icarus, 134, 213

Davies, J. K., Green, S., McBride, N., et al. 2000, Icarus, 146, 253

de Bergh, C., Schmitt, B., Moroz, L. V., Quirico, E., \& Cruikshank, D. P. 2008 in The Solar System Beyond Neptune, eds. M. A. Barucci, H. Boehnhardt, D. P. Cruikshank, A. Morbidelli, \& R. Dotson (U. Arizona Press), 483

Delsanti, A., Hainaut, O., Jourdeuil, E., et al. 2004, A\&A, 417, 1145

Delsanti, A., Peixinho, N., Boehnhardt, H., et al. 2006, AJ, 131, 1851

Delsemme, A. H. 1982, in Comet Discoveries, Statistics, and Observational Selection, ed. L. L. Wilkening, IAU Colloq., 61, 85

Dones, L., Weissman, P. R., Levison, H. F., \& Duncan, M. J. 2004, in Comets II, eds. M. C. Festou, H. U. Keller, \& H. A. Weaver, 153

Doressoundiram, A., Peixinho, N., de Bergh, C., et al. 2002, AJ, 124, 2279

Doressoundiram, A., Boehnhardt, H., Tegler, S. C., \& Trujillo, C. 2008, in The Solar System Beyond Neptune, eds. M. A. Barucci, H. Boehnhardt, D. P. Cruikshank, A. Morbidelli, \& R. Dotson (U. Arizona Press), 91

Dumas, C., Merlin, F., Barucci, M. A., et al. 2007, A\&A, 471, 331

Duncan, M. J., \& Levison, H. F. 1997, Science, 276, 1670

Fulchignoni, M., Belskaya, I., Barucci, M. A., de Sanctis, M. C., \& Doressoundiram, A. 2008, in The Solar System Beyond Neptune, eds. M. A. Barucci, H. Boehnhardt, D. P. Cruikshank, A. Morbidelli, \& R. Dotson (U. Arizona Press), 181

Fulchignoni, M., Birlan, M., \& Barucci, M. A. 2000, Icarus, 146, 204

Gil-Hutton, R., \& Licandro, J. 2001, Icarus, 152, 246

Gladman, B., Marsden, B. G., \& Vanlaerhoven, C. 2008, in The Solar System Beyond Neptune, eds. M. A. Barucci, H. Boehnhardt, D. P. Cruikshank, A. Morbidelli, \& R. Dotson (U. Arizona Press), 43 
Gomes, R. S. 2003, Icarus, 161, 404

Gomes, R. S., Fern Ndez, J. A., Gallardo, T., \& Brunini, A. 2008, in The Solar System Beyond Neptune, eds. M. A. Barucci, H. Boehnhardt, D. P. Cruikshank, A. Morbidelli, \& R. Dotson (U. Arizona Press), 259

Green, S. F., McBride, N., O Ceallaigh, D. P., et al. 1997, MNRAS, 290, 186

Hainaut, O. R., \& Delsanti, A. C. 2002, A\&A, 389, 641 (Paper I)

Hainaut, O. R., Delahodde, C. E., Boehnhardt, H., et al. 2000, A\&A, 356, 1076

Jewitt, D. 2009, AJ, 137, 4296

Jewitt, D. C. 2002, AJ, 123, 1039

Jewitt, D., \& Luu, J. 1998, AJ, 115, 1667

Jewitt, D. C., \& Luu, J. X. 2001, AJ, 122, 2099

Jewitt, D., \& Meech, K. J. 1986, ApJ, 310, 937

Jewitt, D., Peixinho, N., \& Hsieh, H. H. 2007, AJ, 134, 2046

Kavelaars, J., Jones, L., Gladman, B., Parker, J. W., \& Petit, J.-M. 2008, in The Solar System Beyond Neptune, eds. M. A. Barucci, H. Boehnhardt, D. P. Cruikshank, A. Morbidelli, \& R. Dotson (U. Arizona Press), 59

Kenyon, S. J., \& Bromley, B. C. 2004, AJ, 128, 1916

Lacerda, P., Jewitt, D., \& Peixinho, N. 2008, AJ, 135, 1749

Landolt, A. 1992, ApJ, 104, 340

Levison, H. F., \& Stern, S. A. 2001, AJ, 121, 1730

Levison, H. F., Morbidelli, A., Vanlaerhoven, C., Gomes, R., \& Tsiganis, K. 2008, Icarus, 196, 258

Levison, H. F., Duncan, M. J., Brasser, R., \& Kaufmann, D. E. 2010, Science, 329,187

Luu, J. X., \& Jewitt, D. C. 1996, AJ, 111, 499

McKinnon, W. B., Prialnik, D., Stern, S. A., \& Coradini, A. 2008, in The Solar System Beyond Neptune, eds. M. A. Barucci, H. Boehnhardt, D. P. Cruikshank, A. Morbidelli, \& R. Dotson (U. Arizona Press), 213

Meech, K. J., \& Svoren, J. 2004, in Comets II, eds. M. C. Festou, H. U. Keller, \& H. A. Weaver, 317

Meech, K. J., Hainaut, O. R., \& Marsden, B. G. 2004, Icarus, 170, 463

Morbidelli, A., Levison, H. F., Tsiganis, K., \& Gomes, R. 2005, Nature, 435, 462

Morbidelli, A., Levison, H. F., \& Gomes, R. 2008a, in The Solar System Beyond Neptune, eds. M. A. Barucci, H. Boehnhardt, D. P. Cruikshank, A. Morbidelli, \& R. Dotson (Arizona Press), 275
Morbidelli, A., Levison, H. F., \& Gomes, R. 2008b, in The Solar System Beyond Neptune, eds. M. A. Barucci, H. Boehnhardt, D. P. Cruikshank, A. Morbidelli, \& R. Dotson (U. Arizona Press), 275

Müller, T. G., Lellouch, E., Böhnhardt, H., et al. 2009, Earth Moon Planets, 105 , 209

Neese, C. 2011, NASA Planetary Data System, 150

Peixinho, N., Doressoundiram, A., Delsanti, A., et al. 2003, A\&A, 410, L29

Peixinho, N., Lacerda, P., \& Jewitt, D. 2008, AJ, 136, 1837

Perna, D., Barucci, M. A., Fornasier, S., et al. 2010, A\&A, 510, A53

Protopapa, S., Boehnhardt, H., Herbst, T. M., et al. 2008, A\&A, 490, 365

Reyment, R., \& Joreskog, K. 1993 (Cambridge University Press)

Romanishin, W., \& Tegler, S. C. 1999, Nature, 398, 129

Romanishin, W., Tegler, S. C., Levine, J., \& Butler, N. 1997, AJ, 113, 1893

Schaller, E. L. 2010, in Eur. Phys. J. Web Conf., 9, 267

Schaller, E. L., \& Brown, M. E. 2007, ApJ, 659, L61

Sekiguchi, T., Boehnhardt, H., Hainaut, O. R., \& Delahodde, C. E. 2002, A\&A, 385,281

Sheppard, S. S. 2010, AJ, 139, 1394

Sicardy, B., Ortiz, J. L., Assafin, M., et al. 2011, Nature, 478, 493

Stansberry, J., Grundy, W., Brown, M., et al. 2008, in The Solar System Beyond Neptune, eds. M. A. Barucci, H. Boehnhardt, D. P. Cruikshank, A. Morbidelli, \& R. Dotson (U. Arizona Press), 161

Stern, S. A., \& Tholen, D. J. 1998, Pluto and Charon, eds. S. A. Stern, \& D. J. Tholen

Tegler, S. C., \& Romanishin, W. 1998, Nature, 392, 49

Tegler, S. C., \& Romanishin, W. 2000, Nature, 407, 979

Tegler, S. C., \& Romanishin, W. 2003, Icarus, 161, 181

Tegler, S. C., Romanishin, W., \& Consolmagno, S. J. 2003, ApJ, 599, L49

Tegler, S. C., Bauer, J. M., Romanishin, W., \& Peixinho, N. 2008, in The Solar System Beyond Neptune, eds. M. A. Barucci, H. Boehnhardt, D. P. Cruikshank, A. Morbidelli, \& R. Dotson (U. Arizona Press), 161

Tholen, D. J. 1984, Ph.D. Thesis, University of Arizona

Tholen, D. J., \& Barucci, M. A. 1989, in Asteroids II (The University of Arizona Press), 298

Trujillo, C. A., \& Brown, M. E. 2002, ApJ, 566, L125

Trujillo, C. A., Jewitt, D. C., \& Luu, J. X. 2001, AJ, 122, 457

Trujillo, C. A., Sheppard, S. S., \& Schaller, E. L. 2011, ApJ, 730, 105 